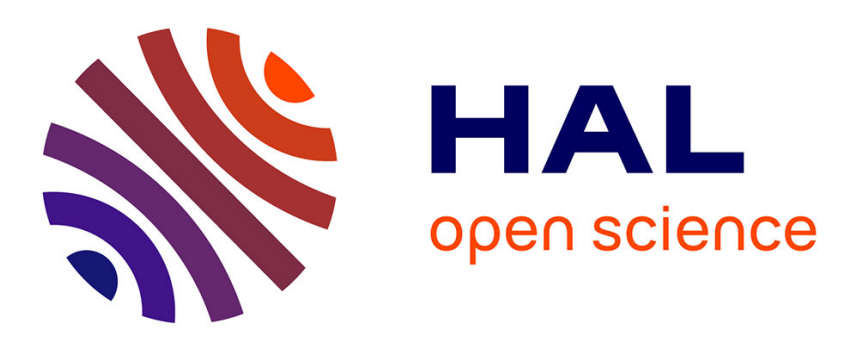

\title{
L'absorption de la vapeur d'eau atmosphérique dans le proche infrarouge (les bandes à 2,7 et $1,8 \mu$ )
}

Romain Mathis

\section{To cite this version:}

Romain Mathis. L'absorption de la vapeur d'eau atmosphérique dans le proche infrarouge (les bandes à 2,7 et 1,8 $\mu$ ). J. Phys. Radium, 1951, 12 (1), pp.51-64. 10.1051/jphysrad:0195100120105100 . jpa-00234344

\section{HAL Id: jpa-00234344 https://hal.science/jpa-00234344}

Submitted on 1 Jan 1951

HAL is a multi-disciplinary open access archive for the deposit and dissemination of scientific research documents, whether they are published or not. The documents may come from teaching and research institutions in France or abroad, or from public or private research centers.
L'archive ouverte pluridisciplinaire HAL, est destinée au dépôt et à la diffusion de documents scientifiques de niveau recherche, publiés ou non, émanant des établissements d'enseignement et de recherche français ou étrangers, des laboratoires publics ou privés. 


\title{
L'ABSORPTION DE LA VAPEUR D'EAU ATMOSPHÉRIQUE DANS LE PROGHE INFRAROUGE (LES BANDES A 2,7 ET 1,8 $\mu$ )
}

\author{
Par M $^{\mathrm{me}}$ R. MATHIS. \\ Laboratoire annexe du Laboratoire de Recherches physiques de la Sorbonne (P. C. B.).
}

\begin{abstract}
Sommaire. - Les bandes de la vapeur d'eau à 2,7 et $1,8 \mu$ ont été étudiées au moyen d'un spectrographe à réseau à cellule au sulfure de plomb. On a essayé d'interpréter une partie des bandes de rotation-vibration obtenues au moyen des transitions permises entre les niveaux d'énergie de la molécule dans les états $(\mathrm{I}, \mathrm{o}, \mathrm{o})(\mathrm{o}, \mathrm{o}, \mathrm{I})(\mathrm{o}, \mathrm{I}, \mathrm{I})$ et l'état fondamental $(0,0,0)$, et l'on a calculé les constantes de rotation dans les trois états $(\mathrm{I}, \mathrm{o}, \mathrm{o})(\mathrm{o}, \mathrm{o}, \mathrm{I})$ et $(0, \mathrm{I}, \mathrm{I})$.
\end{abstract}

L'étude des spectres de rotation pure et de vibration-rotation de la molécule d'eau a permis d'attribuer à celle-ci une structure triangulaire symétrique.

On observe, dans le proche infrarouge, deux fortes bandes d'absorption autour de $\mathrm{I} 595 \mathrm{~cm}^{-1}$ et de $3756 \mathrm{~cm}^{-1}$. La première est attribuée à la vibration de déformation et la seconde à la vibration antisymétrique de valence, on les désigne respectivement par $\nu_{2} \|$ et $\nu_{3} \perp$, les symboles $\|$ et $\perp$ rappelant que dans la vibration $\nu_{2}$ la variation du moment électrique est parallèle à l'axe de symétrie de la molécule, et que dans la vibration $\nu_{3}$ elle lui est perpendiculaire. La vibration symétrique de valence $\left(3652 \mathrm{~cm}^{-1}\right)$ est alors désignée par $\nu_{1} \|$ Elle a été séparée de $\nu_{3}$ récemment par Nielsen.

Nielsen a d'ailleurs introduit une autre nomenclature, qui sera adoptée ici. Une vibration est dite parallèle ou perpendicualire lorsque la variation du moment électrique est parallèle ou perpendiculaire à l'axe de plus petit moment d'inertie de la molécule. Les bandes étudiées dans ce qui suit seront désignées par les symboles

$$
\nu_{1} \perp, \quad v_{3}\left\|, \quad \nu_{2}+v_{3}\right\| .
$$

Dans le problème général du rotateur dans l'espace, le moment angulaire total prend les valeurs quantifiées suivantes :

$$
\sqrt{J(J+1)} \frac{h}{2 \pi} \quad(J \text { entier } \supseteq 0) .
$$

Dans le cas d'un rotateur symétrique, chaque niveau de rotation $J$ se subdivise en $J+$ r sous niveaux caractérisés chacun par un nombre $K$ entier compris entre o et $J$; le niveau $K=$ o, est simple et tous les autres sont doublement dégénérés.

Dans le cas du rotateur asymétrique, auquel se rattache la molécule d'eau, la dégénérescence est levée, et le niveau $J$ se subdivise en $2 J+$ I sous niveaux distincts, qu'on désigne généralement par un indice :

$$
J_{+J}, \quad J_{+J-1}, \quad \ldots, \quad J_{0}, \quad \ldots, \quad J_{-J+1}, \quad J_{-J},
$$

qui n'est pas un nombre quantique, mais un simple numéro. On appelle $J_{+J}$ le sous-niveau le plus élevé et $J_{-J}$ le sous-niveau le moins élevé.

De nombreux auteurs ont étudié le spectre d'absorption infrarouge de la vapeur d'eau. Sleator [1] (г 1 2), Sleator et Phelps [2] (1925), ont obtenu les spectres des bandes de $1,38,1,87,2,66$ et $6,26 \mu$ avec une résolution déjà considérable, mais insuffisante pour l'étude de la structure rotationnelle. Sleator et Plyler [3] ( $193 \mathrm{r}$ ) ont analysé ces mêmes bandes avec une meilleure résolution; ils ont obtenu 3 à 4 fois plus de raies que dans les travaux de 1918 et i925. Bonner [4] (г931) a évalué les constantes d'harmonicité et d'anharmonicité de la molécule.

C'est Mecke [5] qui a le premier réussi l'analyse de la structure rotationnelle de ces bandes. L'interprétation théorique qu'il en a donnée alors reste encore valable aujourd'hui à ceci près qu'il ne tient pas compte de la force centrifuge; on a montré depuis que celle-ci n'a qu'une action très faible tant que $J$ est petit, mais, quand $J$ est élevé, il n'en est plus ainsi; la correction à apporter de ce fait au niveau $3_{3}$ est déjà de l'ordre de $\mathrm{I}^{-1}$ et pour le niveau 44 elle atteint $5 \mathrm{~cm}^{-1}$.

Des progrès considérables ont été faits récemment. Randall, Dennison, Ginsburg et Weber [6] ont enregistré le spectre de rotation pure de la vapeur d'eau donné par un appareil à grande dispersion ( 8 à $75 \mu$ ). A partir des moments d'inertie données par Mecke, ils ont calculé les niveaux de rotation pure jusqu'à $J=\mathrm{II}$. La comparaison entre les niveaux calculés et observés indique des écarts faibles pour les faibles valeurs de $J$, mais qui croissent rapidement avec $J\left(279 \mathrm{~cm}^{-1}\right.$ pour le sous-niveau I I $\left._{11}\right)$. Ces différences peuvent être attribuées principalement à l'effet de la force centrifuge, la molécule d'eau n'étant pas rigoureusement indéformable.

Darling et Dennison [7] ont évalué les moments d'inertie de la molécule et les constantes de la fonctions potentielle. Shaffer et Nielsen [8] ont établi 


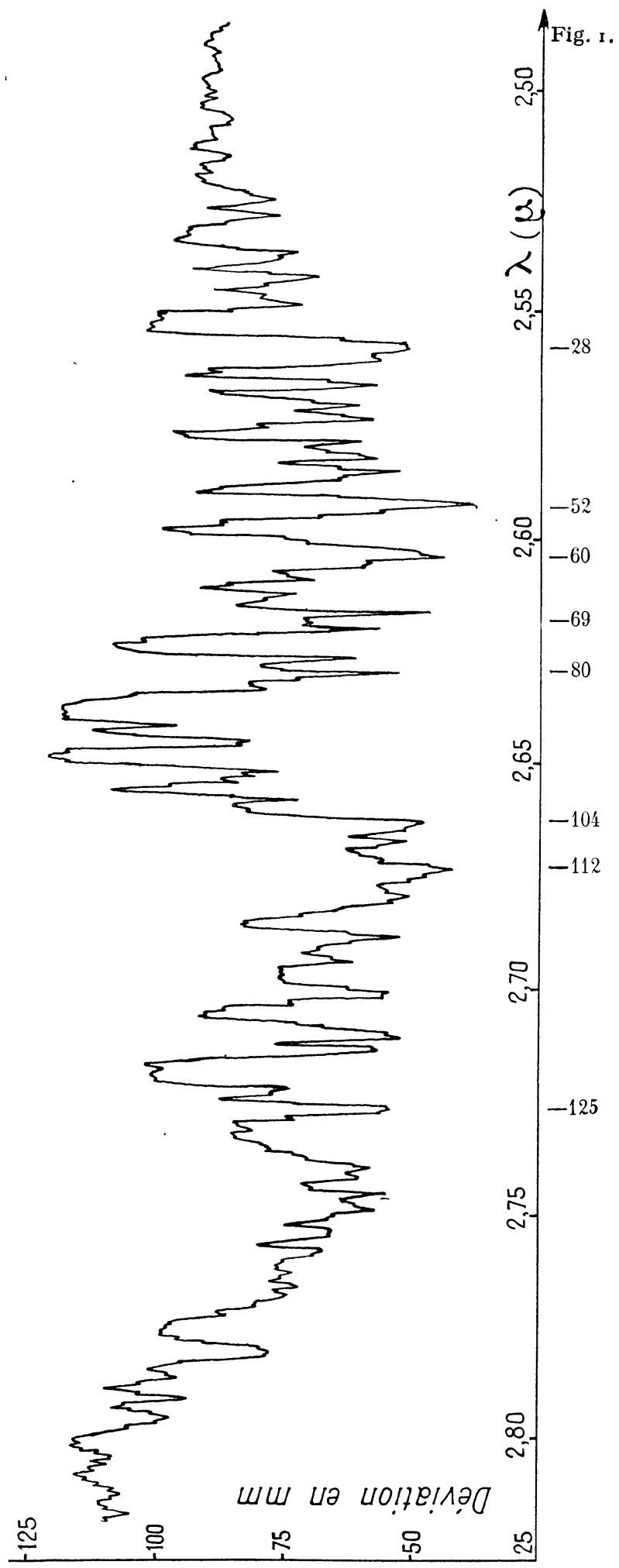

la théorie complète des molécules triatomiques non linéaires, et écrit les équations séculaires qui permettent de déterminer les niveaux d'énergie de

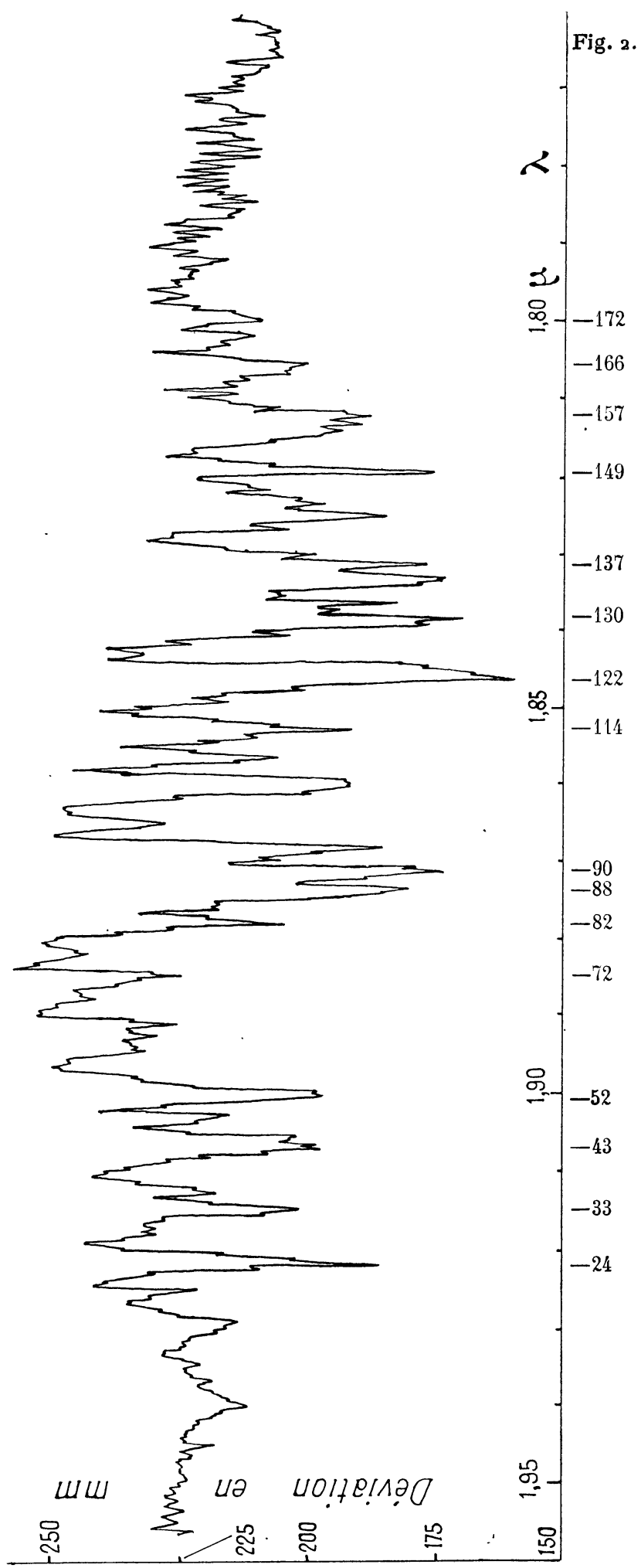

vibration rotation jusqu'à $J=4$; ils tiennent compte de tous les termes provenant de l'interaction entre la vibration et la rotation et de tous ceux pro- 
venant de l'anharmonicité du potentiel, à l'approximation du deuxième ordre. H. H. Nielsen [9, 10] a calculé les niveaux de vibration-rotation jusqu'à $J=6$, pour les bandes $\nu_{2}(6,26 \mu) 2 \nu_{2}(3,3 \mu)$, $\nu_{1}$ et $\nu_{3}(2,7 \mu), \nu_{2}+\nu_{3}(1,87 \mu)$ et il a obtenu d'autre part, pour ces mêmes bandes, des spectres d'une résolution qui n'avait jamais été atteinte auparavant.

Dans le présent travail, l'étude des deux bandes à à 2,7 et $\mathrm{I}, 87 \mu$ a été reprise au moyen d'un spectrographe à réseau; le récepteur est une cellule au sulfure de plomb refroidie par de la neige carbonique. Le signal de cellule est amplifié et commande un dispositif enregistreur. Ce montage a été décrit dans un précédent article [11]. (Les cellules au sulfure de plomb ont déjà été utilisées récemment par différents auteurs, pour l'étude de la structure fine des bandes de vibration-rotation de la molécule d'eau [12, 13, 14]). Le réseau, du type " échelette ", tracé par le professeur Wood, à 8 I 5 traits $/ \mathrm{cm}$, et $7,2 \mathrm{~cm}$ de large. Les fentes d'entrée et de sortie sont ajustées à la même largeur $(0,04 \mathrm{~mm})$; elles occupent, dans le spectre, un domaine de $8 \AA$, ce qui correspond à l'utilisation à peu près totale $\mathrm{du}$ pouvoir de résolution du réseau. Les spectres ont été étalonnés, en identifiant quelques grandes bandes à celles correspondantes de Plyler et Sleator [3].

Bandes $\nu_{1}$ et $\nu_{3}$ à $2,7 \mu$. - Entre les fréquences $3952 \mathrm{~cm}^{-1}$ et 36 or, $6 \mathrm{~cm}^{-1}$, il apparaît r 86 bandes de vibration-rotation ( $f i g$. r), que l'on doit attribuer à la superposition des deux bandes $\nu_{1}$ et $\nu_{3}$ (les deux bandes ne sont pas du même genre et la résonance n'intervient pas). Dans cet intervalle, $H$. $H$. Nielsen a observé i 6 raies qu'il explique par des transitions entre les divers sous-niveaux correspondant à $J=\mathrm{I}$, $2,3,4,5,6$. Il a déterminé ces niveaux, comme il a été indiqué, par un calcul très complet. Ses résultats ont été utilisés pour l'interprétation des bandes que j'ai observées, et j'ai tenté de calculer les niveaux qui correspondent à des valeurs plus élevées de $J$. Le calcul complet est déjà difficile et long pour $J=6$. J'ai déterminé, de $J=7$ à $J=\mathrm{I}$, les sous-niveaux $J_{+J}, \quad J_{J-1}, \quad J_{J-2}, \quad J_{J-3}, \quad J_{-J+3}, \quad J_{-J+2}, \quad J_{-J+1}, \quad J_{-J}$.

Le calcul des niveaux d'énergie de vibration-rotation des molécules rotateurs asymétriques a été fait par Witmer [15], Wang [16], Kramers et Ittmann [17, 18] Klein [19], Ray [20]. Ces auteurs ont donné différentes formules, dont les plus utilisées sont celles de Wang

$$
F\left(J_{\tau}\right)=\frac{\mathrm{I}}{2}(B+C) J(J+\mathrm{I})+\left[A-\frac{\mathrm{I}}{2}(B+C)\right] W_{\tau}
$$

et celle de Ray, corrigée par King, Hainer et Cross [21]

$$
F\left(J_{\tau}\right)=\frac{1}{2}(A+C) J(J+1)+\frac{\mathrm{I}}{2}(A-C) E_{\tau},
$$

où $W_{\tau}$ et $E_{\tau}$ sont des fonctions de $\tau$ et dẹs constantes de rotation

$$
A=\frac{h}{8 \pi^{2} c I_{A}}, \quad B=\frac{h}{8 \pi^{2} c I_{B}}, \quad C=\frac{h}{8 \pi^{2} c I_{C}} .
$$

Pour chaque valeur de $J$, ces fonctions prennent $2 J+\mathrm{I}$ valeurs différentes correspondant chacune à un sous-niveau. Ces $2 J+\mathrm{r}$ valeurs sont les racines de déterminants séculaires de degré $2 J+\mathrm{I}$, dont la résolution est pénible. Toutefois Mecke [5] a montré que, pour une molécule différent peu d'un rotateur symétrique allongé $(B \simeq C)$ pour lequel on aurait $W_{\tau}=K^{2}$, les deux sous-niveaux les plus élevés sont donnés par

$$
\frac{1}{2}\left[F_{J_{+J}}+F_{J_{+J-1}}\right]=A J^{2}+\frac{\mathrm{I}}{2}(B+C) J .
$$

Pour une molécule se rapprochant d'un rotateur symétrique aplati $(B \simeq A)$, ce sont les deux niveaux les plus bas qui sont représentés par

$$
\frac{\mathrm{I}}{2}\left[F_{J_{-J}}+F_{I_{-J+1}}\right]=C J^{2}+\frac{\mathrm{I}}{2}(A+B) \cdot J .
$$

Compte-tenu de la différence entre $W_{\tau}$ et $K^{2}$, Mecke obtint, au lieu de (1) et (2)

$$
\begin{aligned}
& \left.\begin{array}{l}
F_{J_{+J}} \\
F_{J_{J-1}}
\end{array}\right\}=C J^{2}+\frac{\mathrm{I}}{2}(A+B) J \\
& +\frac{\mathrm{I}}{4}(B-A) \cdot J \frac{(2 J-\mathrm{I})}{J-\mathrm{I}} \varepsilon_{C}\left(\mathrm{I}+\varepsilon_{C}^{2}\right), \\
& \left.\begin{array}{l}
F_{J_{-J}} \\
F_{J_{-J+1}}
\end{array}\right\}=A J^{2}+\frac{\mathrm{I}}{2}(B+C) J \\
& +\frac{\mathrm{I}}{4}(B-C) J \frac{(2 J-\mathrm{I})}{J-\mathrm{I}} \varepsilon_{A}\left(\mathrm{I}+\varepsilon_{A}^{2}\right),
\end{aligned}
$$

avec

$$
\begin{aligned}
& \varepsilon_{A}=\frac{\mathrm{I}}{2} \frac{(A-C)-(A-B)}{(A-C)+(A-B)}, \\
& \varepsilon_{C}=\frac{\mathrm{I}}{2} \frac{(C-A)-(C-B)}{(C-A)+(C-B)} .
\end{aligned}
$$

Les équations $\left(1^{\prime}\right)$ et $\left(2^{\prime}\right)$ sont valables avec une bonne approximation pour un rotateur fortement asymétrique, dans le calcul des sous-niveaux

$$
J_{J}, \quad J_{J-1}, \quad J_{-J+1}, \quad J_{-J} .
$$

Des formules analogues peuvent servir au calcul des sous-niveaux $J_{J-2}, J_{J-3}, J_{-J+3}, J_{-J+2}$,

$$
\begin{aligned}
& \left.\begin{array}{l}
F_{-J+2} \\
F_{-J+3}
\end{array}\right\}=C(J-\mathbf{I})^{2}+\frac{\mathbf{I}}{2}(A+B)(3 J-\mathbf{I}) \\
& +\frac{3}{4}(B-A)(J-1) \frac{2 J-1}{J-2} \varepsilon_{C}, \\
& \left.\begin{array}{l}
F_{J-2} \\
F_{J-3}
\end{array}\right\}=A(J-\mathrm{I})^{2}+\frac{\mathrm{I}}{2}(B+C)(3 J-\mathrm{I}) \\
& +\frac{3}{4}(B-C)(J-1) \frac{2 J-1}{J-2} \varepsilon_{A} .
\end{aligned}
$$

Ce sont ces formules qui ont été appliquées à la détermination des sous-niveaux $J_{J}$ à $J_{J_{-3}}$ et $J_{-I+3}$ 
Tableau I.

Les niveaux d'énergie de rotation de la molécule d'eau

(d'après Randall, Dennison, Ginskurg et Weber).

\begin{tabular}{|c|c|c|c|c|c|c|c|}
\hline \multirow{3}{*}{$\begin{array}{l}J \\
\mathrm{o}_{0} \ldots \ldots\end{array}$} & \multicolumn{3}{|l|}{$\nu\left(\mathrm{cm}^{-1}\right)}$. & \multicolumn{2}{|c|}{ J. $\quad \vee\left(\mathrm{cm}^{-1}\right)$} & \\
\hline & o & + & + & $5-4 \ldots$ & 326,49 & - & - \\
\hline & & & & $5_{-5} \ldots$ & 325,23 & - & + \\
\hline $\mathbf{I}_{1} \ldots \ldots$ & 42,30 & + & - & & & & \\
\hline $\mathrm{I}_{0} \ldots \ldots$ & 37,05 & - & - & $6_{6} \ldots$ & I045,34 & + & + \\
\hline $1-1 \ldots$ & 23,78 & - & + & $6_{5} \ldots$ & I045,34 & - & + \\
\hline & & & & $6_{4} \ldots$ & 888,89 & - & - \\
\hline $22 \ldots \ldots$ & I 36, Io & + & + & $6_{3} \ldots$ & 888,85 & + & - \\
\hline $2_{1} \ldots \ldots$ & $\mathrm{I} 34,8 \mathrm{I}$ & - & + & $6_{2} \ldots$ & 757,84 & + & + \\
\hline $2_{0} \ldots \ldots$ & 95,04 & - & - & $6_{1} \ldots$ & $756,9^{\circ}$ & - & + \\
\hline $2-1 \ldots$ & 79,38 & + & - & $6_{0} \ldots$ & 66 I, 63 & - & - \\
\hline $2-2 \ldots$ & 70,03 & + & + & $6_{-1} \ldots$ & 649, I I & + & - \\
\hline & & & & $6_{-2} \ldots$ & 602,78 & + & + \\
\hline $3_{3} \ldots$ & 285,45 & + & - & $6-_{-3} \ldots$ & 553, oo & - & + \\
\hline $3_{2} \ldots \ldots$ & 285,26 & - & - & $6 \_-4$ & 542,80 & - & - \\
\hline $3_{1} \ldots \ldots$ & 212,07 & - & + & $6-5 \ldots$ & 447,20 & + & - \\
\hline $3_{0} \ldots$ & 206,25 & + & + & $6_{-6} \ldots$ & 446,69 & + & + \\
\hline $3-1 \ldots$ & $\mathbf{I} 73,33$ & + & - & & & & \\
\hline $3-2 \ldots$ & $\mathrm{I} 42, \mathrm{I} 7$ & - & - & $7_{7} \ldots$ & I 395,25 & + & - \\
\hline $3_{-3} \ldots$ & 1 36,74 & - & + & $7_{6} \ldots$ & I 395,26 & -- & - \\
\hline & & & & $7_{5} \ldots$ & І 2 I 6,63 & - & + \\
\hline $44 \ldots$ & 488,24 & + & + & $7+\ldots$ & г 2 І 6,63 & + & $-\ldots$ \\
\hline $43 \ldots \ldots$ & 488,24 & - & + & $7_{3} \ldots$ & 1060,07 & $-t$ & - \\
\hline $42 \ldots \ldots$ & $383,9^{\circ}$ & - & - & $7_{2} \ldots$ & I059,88 & - & - \\
\hline $41 \ldots$ & 382,49 & + & - & $71 \ldots$ & $9^{3}$ г, 50 & - & -. \\
\hline $40 \ldots$ & 3 I 5,66 & + & + & $7_{0} \ldots$ & 927,92 & + & + \\
\hline $4-1 \ldots$ & 300,33 & - & + & $7-1 \ldots$ & 842,65 & - & - \\
\hline $4-2 \ldots$ & $275,2 \mathrm{I}$ & - & - & $7-2 \ldots$ & 816,78 & - & - \\
\hline $4-3 \ldots$ & 224,74 & + & - & $7-3 \ldots$ & 782,54 & - & + \\
\hline $4-i \ldots$ & $22 \mathrm{I}, 90$ & + & + & $7-i \ldots$ & 709,54 & -+ & $-i-$ \\
\hline & & & & $7-5 \cdots$ & 704,40 & + & - \\
\hline $5_{5} \ldots \ldots$ & 742,30 & + & - & $7-6 \cdots$ & 586,53 & - & - \\
\hline $5, \ldots \ldots$ & 742,30 & - & - & $7-7 \ldots$ & 586,32 & - & + \\
\hline $5_{3} \ldots \ldots$ & 610,42 & - & + & & & & \\
\hline $5_{2} \ldots$ & 6 Io, 42 & + & + & $8_{8} \ldots$ & I 789,77 & + & + \\
\hline $5, \ldots \ldots$ & 508,86 & + & - & $8_{7} \ldots$ & 1789,77 & - & + \\
\hline $5_{0} \ldots \ldots$ & 503,94 & - & - & $8_{6} \ldots$ & 1591,47 & - & - \\
\hline $5_{-1} \ldots$ & 446,52 & - & + & $8_{5} \ldots$ & I59I, 47 & + & - \\
\hline $5-2 \ldots$ & 416,02 & + & + & $8_{4} \ldots$ & $\mathrm{I} 4 \mathrm{II}, 89$ & + & + \\
\hline $5-3 \ldots$ & 399,45 & + & - & $8_{3} \ldots$ & $\mathrm{I} 4 \mathrm{II}, 89$ & - & + \\
\hline
\end{tabular}

à $J_{-J}$, pour les valeurs de $J$ allant de o à I I. Nous avons utilisé les valeurs des moments d'inertie indiquées par Darling et Dennison [7]. Les résultats de ces calculs pour $J=1,2, \ldots, 6$, diffèrent de ceux de Nielsen, et ceci d'autant plus que $J$ est plus grand. Cet écart doit être attribué au fait que, dans les formules $\left(1^{\prime}\right),\left(2^{\prime}\right)$, $\left(3^{\prime}\right)$ et $\left(4^{\prime}\right)$, il n'est pas tenu compte de la force centrifuge. La correction nécessaire a été faite, en admettant que, pour un niveau quelconque de vibration-rotation, la différence entre le niveau calculé et le niveau exact est la même qu'entre le niveau correspondant de rotation pure calculé et le niveau observé par Randall

\begin{tabular}{|c|c|c|c|c|c|c|c|}
\hline$J$. & $\nu\left(\mathbf{c m}^{-1}\right)$ & & & $J$. & $\nu\left(\mathrm{cm}^{-1}\right)$ & & \\
\hline $8_{2} \ldots \ldots$ & I256, 24 & - & - & $\mathrm{IO}_{3} \ldots \ldots$ & 2055,04 & + & - \\
\hline $8_{1} \ldots$ & I 255,45 & + & - & $\mathrm{IO}_{2} \ldots \ldots$ & I 876, I 6 & + & + \\
\hline $8_{0} \ldots \ldots$ & I I 32, Io & + & + & $\mathrm{IO}_{1} \ldots \ldots$ & $\mathrm{I} 875,68$ & - & + \\
\hline $8-1 \ldots$ & I I 23, I I & + & + & $\mathrm{IO}_{0} \ldots \ldots$ & 1726,23 & - & - \\
\hline $8-2 \ldots$ & I052,92 & - & - & $1 O_{-1} \ldots$ & 1719,75 & + & - \\
\hline $8-3 \ldots$ & เ 006,38 & + & - & IO-2 $\ldots$ & 1614,43 & + & + \\
\hline $8-4 \ldots$ & $9^{83,22}$ & + & + & IO & I 521,88 & - & + \\
\hline $8_{-5} \ldots$ & 885,84 & - & + & $10-4 \ldots$ & I 538,65 & -- & - \\
\hline $8_{-6} \ldots$ & 883, I 2 & - & - & I $\mathrm{O}_{-5} \ldots$ & I 446,68 & + & - \\
\hline $8_{-7} \ldots$ & 744,31 & + & - & $1 O_{-6} \ldots$ & I 438,50 & + & + \\
\hline $8_{-8} \ldots$ & 744,24 & + & + & $\mathrm{IO}_{-7} \ldots$ & 1294,07 & - & + \\
\hline & & & & IO_s $\ldots$ & I 293,49 & - & - \\
\hline $9, \ldots \ldots$ & 2226,41 & + & - & ${ }^{I} O_{-9} \ldots$ & I I I 4,9 I & + & - \\
\hline $98 \ldots$ & $2226,4 \mathrm{I}$ & - & - & IO_-10 $\ldots$ & I I I 4,9 I & + & + \\
\hline $97 \ldots \ldots$ & 2010,67 & - & + & & & & \\
\hline $9_{6} \ldots \ldots$ & 2010,67 & + & + & $\mathrm{II}_{11} \ldots \ldots$ & 3218,55 & + & - \\
\hline $9_{5} \ldots \ldots$ & $\mathrm{I} 8 \mathrm{I} \mathrm{I}, \mathrm{I} 8$ & + & - & $\mathrm{II}_{10} \ldots \ldots$ & $32 \mathrm{I} 8,55$ & - & - \\
\hline $9_{4} \ldots \ldots$ & I 8 II, I 8 & - & - & $\mathrm{I}_{9} \ldots \ldots$ & 2974,88 & - & + \\
\hline $93 \ldots \ldots$ & I63 I , 95 & - & + & $\mathbf{I} \mathbf{I}_{8} \ldots \ldots$ & 2974,88 & + & + \\
\hline $92 \ldots$ & I 63 I, 8 I & + & + & $\mathrm{II}_{7} \ldots \ldots$ & 2741,42 & + & - \\
\hline $9_{1} \ldots \ldots$ & 1477,78 & + & - & $\mathrm{II}_{6} \ldots \ldots$ & 2741,42 & - & - \\
\hline $9_{0} \ldots$ & $\mathbf{1} 475,46$ & - & - & $\mathrm{I}_{\mathrm{I}_{5}} \ldots \ldots$ & 2523,08 & - & + \\
\hline $9-1 \ldots$ & I 360,85 & - & + & $\mathrm{II}_{4} \ldots \ldots$ & 2523,08 & + & + \\
\hline $9-2 \ldots$ & I340, 98 & + & + & $\mathrm{II}_{3} \ldots \ldots$ & 2322,82 & + & - \\
\hline $9-3 \ldots$ & I 283,29 & + & -- & $\mathbf{I}_{2} \mathbf{I}_{2} \ldots$ & 2322,77 & - & - \\
\hline $9-4 \ldots$ & I 2 I 6,62 & - & -- & $\mathrm{I}_{1} \ldots \ldots$ & 2145,17 & - & + \\
\hline $9-5 \ldots$ & I 202,28 & - & + & $\mathrm{II}_{0} \ldots \ldots$ & $2 \mathrm{I} 44,06$ & + & + \\
\hline $9-6 \ldots$ & 1080,72 & + & + & $\mathrm{I}_{-1} \ldots$ & $2000,7 \mathrm{I}$ & + & - \\
\hline $9-7 \ldots$ & $1079,4 \mathrm{I}$ & + & - & $I_{1} I_{-2} \ldots$ & I 984,20 & - & - \\
\hline $9-8 \ldots$ & $9^{20}, 4 \mathrm{I}$ & - & - & I I $-3 \ldots$ & I 898,56 & - & + \\
\hline \multirow[t]{2}{*}{$9-9 \ldots$} & $920,4 \mathrm{I}$ & - & + & I I $-4 \ldots$ & I 840, I 4 & + & + \\
\hline & & & & $I_{I}-5 \ldots$ & I 8 I I , 2 I & + & - \\
\hline$o_{10} \ldots \ldots$ & 2703,28 & + & + & $I I_{-6} \ldots$ & I694,7I & - & - \\
\hline Io $0_{9} \ldots$ & 2703,28 & - & + & II $-7 \ldots$ & $\mathrm{I} 69 \mathrm{I}, \mathrm{I} 3$ & - & + \\
\hline $\mathrm{IO}_{8} \ldots \ldots$ & 2472,44 & - & - & I I_-8 $\ldots$ & I 525,65 & + & + \\
\hline $\mathrm{Io}_{7} \ldots \ldots$ & 2472,44 & + & - & $I_{I} I_{-9} \ldots$ & $\mathbf{I} 525,36$ & + & - \\
\hline$o_{6} \ldots \ldots$ & 2255,08 & + & + & I I $-10 \ldots$ & $\mathrm{I} 327,58$ & - & - \\
\hline $\mathrm{o}_{5} \ldots \ldots$ & 2255,08 & - & + & $I_{1} I_{-11} \ldots$ & I 327,58 & & + \\
\hline
\end{tabular}

et ses collaborateurs dans le travail cité plus haut. Il suffit donc d'ajouter aux valeurs calculées au moyen des formules $\left(1^{\prime}\right),\left(2^{\prime}\right),(3)$ et $\left(4^{\prime}\right)$ la différence en question.

L'accord entre les résultats de cette méthode et ceux de Nielsen pour les six premières valeurs de $J$ donne à penser qu'elle reste encore valable pour des valeurs de $J$ plus élevées.

Le tableau I donne les valeurs des niveaux de rotation pure donnés par Randall, Dennison, Ginskurg et Weber, et le tableau II les divers sousniveaux des états de vibration $\nu_{1}(\mathrm{I}, \mathrm{O}, \mathrm{o})$, et $\nu_{3}(\mathrm{O}, \mathrm{O}, \mathrm{I})$ Par soustraction, on obtient les fréquences des 
Tableau II.

\begin{tabular}{|c|c|c|c|c|c|c|c|}
\hline$J$ & $v_{1}\left(\mathbf{c m}^{-1}\right)$. & & $\nu_{3}\left(\mathrm{~cm}^{-1}\right)$. & $J$ & $\nu_{1}\left(\mathrm{~cm}^{-1}\right)$ & $\nu_{3}\left(\mathrm{~cm}^{-1}\right)$. & \\
\hline $0 \ldots$ & 3652,7 & ++ & $3756,8++$ & $\begin{array}{l}5_{0} \ldots \\
5_{-1} \ldots\end{array}$ & $\begin{array}{l}4132, I-- \\
4075,5-+\end{array}$ & & $1-$ \\
\hline $1 \ldots \ldots$ & 3694,5 & +- & $3797,3+-$ & $5-2 \ldots$ & $4,2++$ &, 9 & $9+$ \\
\hline$I_{0} \ldots$ & 3689,8 & $-\cdots$ & $379^{2}, \mathrm{I}--$ & $-3 \ldots$ & $4035,2 \div-$ & & $7+-$ \\
\hline$-1 \ldots$ & $3677, o$ & -+ & $3779,8-+$ & $-5 \ldots$ & $\begin{array}{l}3,2-- \\
, 5-+\end{array}$ & & - \\
\hline $2 \cdots$ & 3780,6 & ++ & $3887,2+\perp$ & & & & \\
\hline $1 \ldots$ & 377 & -+ &, $2-+$ & ${ }_{6} \ldots \ldots$ &, $9 \div$ &, 9 & ++ \\
\hline $0 \ldots$ & 3740,7 & $-\cdots$ & $3850,2--$ & $\ldots \ldots$ & $9-+$ & & -+ \\
\hline$-1 \ldots$ & 372 & +- & $3833,8+-$ & $4 \ldots \ldots$ & $3-\cdots$ & & - \\
\hline$-2 \ldots$ & 371 & ++ & $3826,2++$ & $\begin{array}{l}3 \ldots \\
\ldots \ldots\end{array}$ & $\begin{array}{l}5+- \\
5++\end{array}$ & & $\begin{array}{l}+- \\
++\end{array}$ \\
\hline $3 \ldots$ & 3 & +- & $4027,1+-$ & $b_{1} \ldots \ldots$ &, $7-+$ & & -1 \\
\hline $2 \ldots$ & $39^{3}$ & -- & $I-\cdots$ & & $8--$ & & $-\cdots$ \\
\hline $1 \ldots$. & 385 & -+ & $8-+$ & $-1 \ldots$ & $7--$ & & +- \\
\hline${ }_{0} \ldots$. & 3852 & ++ & $39^{5} 7,2++$ & $-2 \ldots$ & $5++$ & & ++ \\
\hline$-1 \ldots$ & 3820,2 & +- & $4+-$ & $-3 \ldots$ & $8-+$ & & -+ \\
\hline$-2 \ldots$ & 378 & -- & $I--$ & $-1 \ldots$ & $I \ldots$ & & -- \\
\hline$-3 \cdot \ldots$ & 378 & -+ & $3891,7-+$ & $\begin{array}{l}6_{-5} \ldots \\
6-6 \cdots\end{array}$ & $\begin{array}{l}, 5+- \\
, 5++\end{array}$ & & $\begin{array}{l}+- \\
++\end{array}$ \\
\hline . & & ++ & $4++$ & & & & \\
\hline $3 \cdots$ & 412 & $\cdots+$ & $0-+$ & & $5+-$ & & +- \\
\hline $2 \ldots$ & 402 & -- & $4 \mathrm{II} 3,2--$ & $6 \cdots \cdots$ & $5--$ &, 5 & -- \\
\hline$\ldots$ & $4023, I$ & +- & $4 \mathrm{II} 2,0+-$ & $\ldots$ &, $5-\cdots$ & 49 & $\ldots$ \\
\hline . & $396 \mathrm{r}, 2$ & ++ & $4066,8++$ & $7_{4} \ldots$ & $4825,5++$ & 4925 & +1 \\
\hline . & $3952, I$ & -+ & $4051,2 \ldots$ & . & $\cdots$ & & $\cdots$ \\
\hline-2 & 392 & -- & $4--$ & $7-4 \ldots$ & $4++$ &, 2 & $\div-$ \\
\hline-3 . & 3873,2 & +- & $3978, o+-$ & . & $4+-$ &, 2 & $-\cdots$ \\
\hline 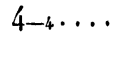 & $387 \mathrm{I}, \mathrm{I}$ & ++ & $3975,5++$ & $\begin{array}{l}7-6 \ldots \\
7-7 \ldots\end{array}$ & $\begin{array}{l}, 8-- \\
, 8-+\end{array}$ & $\begin{array}{l}4334,6 \\
4334,6\end{array}$ & -- \\
\hline & - & 7 & $4+-$ & & & & \\
\hline . & - & 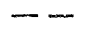 & $4463,4--$ & & $5402,3+\cdots$ &, 4 & ++ \\
\hline & - & & $4345,9-+$ & & $5402,3-+$ & & -+ \\
\hline ra & 4242 , & & $4345,9+-$ & & $5208,1--$ & 6 & -- \\
\hline . & 4141 & & $4249,7-$ & b. &, $\mathrm{I}+-$ & 2,0 & 一 \\
\hline
\end{tabular}

\begin{tabular}{|c|c|c|}
\hline$J$. & $v_{1}\left(\mathrm{~cm}^{-1}\right)$. & $v_{3}\left(\mathrm{~cm}^{-1}\right)$. \\
\hline & 5236 & (1) \\
\hline $\begin{array}{l}\delta_{-5} \ldots \\
8_{-6} \ldots\end{array}$ & $4523,6-$ & $4827,7-$ \\
\hline $8_{-7} \ldots$ & $4379,7+\cdots$ & $449^{\circ}, 1+$ \\
\hline $8_{-8} \ldots$ & $4379,7++$ & $4490,1++$ \\
\hline $99 \ldots \ldots$ & $5827,8+-$ & $5876,8+$ \\
\hline $98 \ldots$ & $5827,8--$ & $5876,8--$ \\
\hline $97 \ldots$ & $5618,1-+$ & $568 \mathrm{I}, 7-+$ \\
\hline $9_{0} \ldots$ & $56 \mathrm{I} 8, \mathrm{I}++$ & $568 \mathrm{I}, 7++$ \\
\hline$\ldots \ldots$ & $\cdots \cdots$ & $\ldots$. \\
\hline $9-6 \cdot \cdots$ & $4715,6-+$ & $4820,2++$ \\
\hline $9-7 \ldots$ & $4715,6+-$ & $4820,2+$ \\
\hline $9-8 \ldots$ & $4555,2 \cdots$ & $4663,6--$ \\
\hline$-9 \cdots$ & $4555,2-+$ & $4663,6-+$ \\
\hline $10 \ldots$ & 63 о, $9++$ &, $8+$ \\
\hline $9 \ldots$ & $6301,9-+$ & $6328,8-+$ \\
\hline $\mathrm{o}_{8} \ldots \ldots$ & $6068,8-\cdots$ & $6 \mathrm{I} 2 \mathrm{I}, 4$ \\
\hline $7 \ldots$ & $6068,8+-$ & $612 \mathrm{I}, 4$ \\
\hline & & - \\
\hline$-7 \ldots$ & $4925,5-+$ & 5030,6 \\
\hline$-8 \ldots$ & $4925,5--$ & $5030,6--$ \\
\hline$-9 \ldots$ & $4745,9+-$ & $4855,2+-$ \\
\hline$-10 \cdots$ & $4745,9++$ & $4855,2+$ \\
\hline $\mathbf{I}_{11} \ldots \ldots$ &, $2+-$ & $6+$ \\
\hline$I_{10} \ldots \ldots$ & $6795,2 \cdots \cdots$ & 68 I6,6 - - \\
\hline $9 \ldots$ & $6538,9-+$ & $6593,0-+$ \\
\hline$I_{8} \ldots \ldots$ & $6538,9++$ & $6593,0++$ \\
\hline & . $\cdots .$. & $5-2-$ \\
\hline-8 & $52.53,2++$ & $53 \mathrm{I}$ \\
\hline$I-9 \ldots$ & $5253,1+-$ & $5316,4+$ \\
\hline$I-10$. & $4954,0--$ & $5064,9-$ \\
\hline & ( & 5 \\
\hline
\end{tabular}

De $J=0$ à $J=6$, les niveaux d'énergie indiqués sont ceux calculés par Nielsen.

composantes des bandes $\nu_{1}[(\mathrm{I}, \mathrm{o}, \mathrm{o})-(\mathrm{o}, \mathrm{o}, \mathrm{o}, \mathrm{I}]$ et $\left.\left.\nu_{3}[0,0,1)-\mathrm{I}_{0}, 0,0\right)\right]$. La règle de sélection bien connue n'autorise que les transitions pour lesquelles $\Delta J=o$ et $\Delta J= \pm \mathrm{I}$. Mais les transitions entre des sous-niveaux quelconques de deux niveaux de vibration-rotation $\left(v_{1}^{\prime}, v_{2}^{\prime}, v_{3}^{\prime}, J^{\prime}\right.$ et $\left.v_{1}^{\prime \prime}, v_{2}^{\prime \prime}, v_{3}^{\prime \prime}, J^{\prime \prime}\right)$ donnés ne sont pas toutes permises. Chaque sousniveau peut-être affecté d'un double signe (cf. Herzberg) qui rend compte de ses propriétés de symétrie, et Dennison [22] a montré que seules sont permises les transitions symbolisées par

et par

$$
\left.\begin{array}{l}
++\leftrightarrow-- \\
+-\leftrightarrow-+
\end{array}\right\} \text { pour } v_{1}
$$$$
\begin{aligned}
& ++\leftrightarrow-+\} \text { pour } v_{2} . \\
& +-\leftrightarrow-\infty
\end{aligned}
$$

La comparaison avec les fréquences expérimentales est indiquée tableau III. Les fréquences marquées d'un astérisque sont celles relatives à la bande $\nu_{1}$.

Bande $\nu_{2}+\nu_{3}$ à $1,87 \mu$. - Un travail analogue a été fait pour cette bande. Le tableau IV indique les valeurs des énergies des sous-niveaux, de $J=\mathrm{I}$ à $J=\mathrm{I}$. Les fréquences calculées et les fréquences observées de cette bande sont rassemblées dans le tableau V [ I 83 bandes de vibration-rotation ont été observées pour cette bande de 5 I 5 I, 4 à $5556,2 \mathrm{~cm}^{-1}$ (fig. 2)]. Les transitions permises pour cette bande sont symbolisées par

$$
\begin{aligned}
& ++\leftrightarrow-+1 v_{2}+v_{3} . \\
& +-\leftrightarrow-1 v^{-}
\end{aligned}
$$


Tableau III.

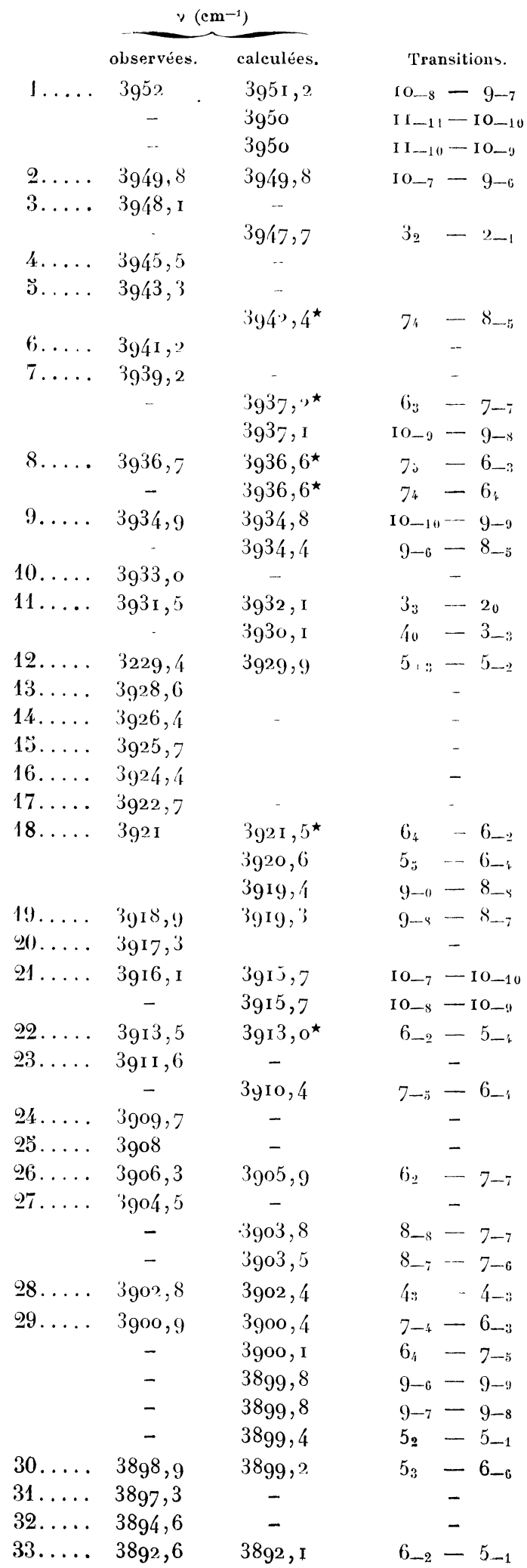

\begin{tabular}{|c|c|c|c|c|c|}
\hline & \multicolumn{2}{|c|}{$y\left(\mathrm{~cm}^{-1}\right)$} & \\
\hline & observées. & \multirow{2}{*}{$\begin{array}{c}\text { calculées. } \\
-\end{array}$} & & & \\
\hline & 3890,7 & & \multicolumn{3}{|c|}{$\begin{array}{c}\text { Transitions. } \\
\text { - }\end{array}$} \\
\hline \multirow{2}{*}{$34 \ldots \ldots$} & - & 3889,7 & $\mathrm{II}_{8}$ & -1 & $\mathrm{IO}_{9}$ \\
\hline & - & 3889,7 & $\mathrm{II}_{-8}$ & $-I$ & $\mathrm{O}_{-7}$ \\
\hline \multirow[t]{2}{*}{$3 \check{3} \ldots .}$. & 3888,2 & 3887,9 & $7-7$ & - & $6-6$ \\
\hline & - & 3887 , 禾 & $7-6$ & - & $6-5$ \\
\hline $36 \ldots$ & 3886,3 & $3886,4^{\star}$ & 41 & - & $3-3$ \\
\hline $37 \ldots$ & $3884, \mathrm{I}$ & - & & - & \\
\hline \multirow[t]{3}{*}{$38 \ldots$} & $388 \mathbf{r}, 9$ & $388 \mathrm{I}, 8$ & 6.2 & $\ldots$ & $5_{3}$ \\
\hline & - & $388 \mathbf{1}, 8$ & $6-1$ & - & $5_{0}$ \\
\hline & - & $388 \mathrm{I}, \mathrm{s}^{\star}$ & $6_{1}$ & - & $5_{1}$ \\
\hline \multirow{3}{*}{$39 \ldots$} & 3880,6 & - & & - & \\
\hline & - & 3879,7 & 74 & - & 6 \\
\hline & - & 3879,7 & $7:$ & - & $6_{6}$ \\
\hline $10 \ldots$. & 3878,6 & - & & - & \\
\hline \multirow[t]{2}{*}{$41 \ldots$} & 3876,5 & - & & - & \\
\hline & - & 3875,5 & 5 & - & 1-2 \\
\hline $42 \ldots$ & 3874,5 & - & & - & \\
\hline $43 \ldots \ldots$ & 3872,7 & - & & - & \\
\hline \multirow[t]{3}{*}{ 4k.... } & 3871,2 & 3871 & $6-6$ & - & 5 \\
\hline & - & 3870,7 & & - & \\
\hline & - & 3870,3 & $i_{-i}$ & - & $5-4$ \\
\hline 保..... & 3869,6 & - & & - & \\
\hline \multirow[t]{4}{*}{$40 \ldots$} & $3866 ;, 7$ & 3866,8 & $7-4$ & - & $7-7$ \\
\hline & - & 3866,8 & $7-5$ & - & $7-5$ \\
\hline & - & 3865,8 & $\tilde{J}_{1}$ & - & 42 \\
\hline & - & 3865,6 & $5-2$ & - & 1-1 \\
\hline $47 \ldots$ & 3863,6 & 3863,4 & $? 2$ & - & $I_{1}$ \\
\hline 18.... & 3862,6 & - & & - & \\
\hline \multirow[t]{2}{*}{ 19.... } & 3859,8 & - & & - & \\
\hline & - & 3859,0 & $5-5$ & - & 1-4 \\
\hline \multirow[t]{4}{*}{$80 \ldots$} & 3858,3 & $3858, \mathrm{I}^{\star}$ & $f_{6}$ & - & $7-2$ \\
\hline & - & 3857,7 & $5-i$ & - & $3-3$ \\
\hline & - & 3857,7 & $5_{2}$ & - & 43 \\
\hline & - & 3857,7 & $5_{3}$ & - & $4 i$ \\
\hline \multirow[t]{2}{*}{$\ddot{31} \ldots .}$. & 3856,6 & - & & - & \\
\hline & - & $3855, \mathrm{I}$ & $4-2$ & - & $3-1$ \\
\hline \multirow[t]{3}{*}{$.2 \ldots$} & 3854,7 & 3854,7 & $6 \ldots-3$ & - & $6-6$ \\
\hline & - & 3854,7 & $1 / 10$ & - & $3_{1}$ \\
\hline & - & 3853,8 & & - & \\
\hline $5: 3 \ldots$ & 3852,8 & 3852,6 & $5_{0}$ & - & 41 \\
\hline \multirow[t]{2}{*}{$\dddot{3} 4 \ldots}$. & $385 \mathrm{r}, 3$ & $3850,8^{\star}$ & $5-1$ & - & $4-3$ \\
\hline & - & 3850,2 & 6 -i & - & $6-5$ \\
\hline ร็..... & 3849,4 & - & & - & \\
\hline $56 \ldots$. & 3846,6 & $3846, \mathrm{I}^{\star}$ & $10_{-7}$ & - & $9-7$ \\
\hline \multirow[t]{2}{*}{$87 \ldots}$. & 3844,8 & 3844,8 & $4-1$ & - & $3_{0}$ \\
\hline & - & $3844,9^{\star}$ & $10-8$ & - & $9-6$ \\
\hline \multirow[t]{2}{*}{$38 \ldots}$. & 3843,2 & - & & - & \\
\hline & - & $3842,2^{\star}$ & $6-1$ & - & $5-1$ \\
\hline $59 \ldots \ldots$ & 3841,7 & $384 \mathrm{I}, 8^{\star}$ & $4:$ & - & $3_{2}$ \\
\hline & - & $384 \mathrm{I}, 6^{\star}$ & $4: 3$ & - & $3:$ \\
\hline & - & $384 \mathbf{I}, 3^{\star}$ & $5_{1}$ & - & $4-1$ \\
\hline
\end{tabular}


Tableau III (suite).

\begin{tabular}{|c|c|c|c|c|}
\hline & \multicolumn{2}{|c|}{$\nu\left(\mathrm{cm}^{-1}\right)$} & \multirow{2}{*}{\multicolumn{2}{|c|}{ Transitions. }} \\
\hline & observées. & calculées. & & \\
\hline \multirow[t]{3}{*}{$60 \ldots}$. & $38 / 10,2$ & 3840,7 & $5-2$ & $-5_{-\ddot{a}}$ \\
\hline & - & $3839, \mathrm{r}^{\star}$ & I I-11 & $-\mathrm{IO}-9$ \\
\hline & - & $3839, \mathrm{r}^{\star}$ & I I -10 & $-10-10$ \\
\hline \multirow[t]{3}{*}{$61 \ldots$} & 3838,3 & 3838,8 & $4-4$ & - 3_: \\
\hline & - & 3835,8 & $4-3$ & $-3-2$ \\
\hline & - & 3835,6 & $5_{0}$ & $-5-3$ \\
\hline $62 \ldots$. & 3834,9 & - & & - \\
\hline \multirow[t]{4}{*}{$63 \ldots$} & 3833,3 & - & & - \\
\hline & - & $3832,5^{\star}$ & $9-6$ & $-8_{-6}$ \\
\hline & - & 3832,4 & $3-1$ & $-2_{0}$ \\
\hline & - & $3832,2^{\star}$ & $6_{5}$ & $-7-1$ \\
\hline \multirow[t]{2}{*}{$64 \ldots$} & $383 \mathrm{r}, 5$ & - & & - \\
\hline & - & $3829,8^{\star}$ & $9-7$ & $-8_{-\ddot{t}}$ \\
\hline \multirow[t]{2}{*}{$65 \ldots$} & $3829, \mathrm{I}$ & $3829,4^{\star}$ & $1 O_{10}$ & $-\mathrm{IO}_{8}$ \\
\hline & - & $3829,4^{\star}$ & $\mathrm{IO}_{4}$ & $-\mathrm{IO}_{7}$ \\
\hline \multirow[t]{4}{*}{$66 \ldots \ldots$} & 3828,3 & $3828,3^{\star}$ & $9_{G}$ & $-8_{7}$ \\
\hline & - & $3828,3^{\star}$ & 97 & $-8_{8}$ \\
\hline & - & 3827,7 & $3_{1}$ & -22 \\
\hline & - & 3826,7 & 41 & $-3_{2}$ \\
\hline \multirow[t]{2}{*}{$67 \ldots$} & 3825,8 & $38 \mathbf{2} 5,5^{\star}$ & IO_-9 & $-9-9$ \\
\hline & - & $3825,5^{\star}$ & IO-10 & $-9-8$ \\
\hline \multirow[t]{2}{*}{$68 \ldots$} & $3824, \mathrm{I}$ & 3824,2 & $5-3$ & -5 \\
\hline & - & $3823, o$ & $6 s$ & $-7_{0}$ \\
\hline \multirow[t]{3}{*}{$69 \ldots \ldots$} & $3822, \mathrm{I}$ & 3822,4 & $3_{0}$ & $-2_{1}$ \\
\hline & - & $3821,9^{\star}$ & 42 & $-3_{0}$ \\
\hline & - & 3821,7 & $3-3$ & - 2_-2 \\
\hline \multirow[t]{4}{*}{$70 \ldots$} & 3820,4 & 3820,5 & $3_{0}$ & $-3_{-3}$ \\
\hline & - & 3819,4 & $6_{6}$ & $-7_{1}$ \\
\hline & - & $38 \mathbf{1} 9,2^{\star}$ & $8-5$ & $-7-5$ \\
\hline & - & $3819,0^{\star}$ & 40 & $-3_{-2}$ \\
\hline $71 \ldots$ & 38 I 8,6 & - & & - \\
\hline \multirow[t]{5}{*}{$72 \ldots \ldots$} & 3817,5 & $3817, \mathrm{I}^{\star}$ & $9_{s}$ & $-9 \mathrm{c}$ \\
\hline & - & $3817, \mathbf{I}^{\star}$ & $9_{9}$ & $-9^{7}$ \\
\hline & $\tau$ & $38 \div 6,7$ & $3-2$ & $-2-1$ \\
\hline & - & $38 \mathrm{r} 6,4^{\star}$ & $5_{1}$ & $-5_{-5}$ \\
\hline & - & $38 \mathrm{I} 6,4^{\star}$ & $5_{0}$ & -40 \\
\hline \multirow[t]{2}{*}{$73 \ldots$} & $38 \mathbf{1 5}, 6$ & $38 \mathbf{1} 6,2$ & 21 & $-2_{-2}$ \\
\hline & - & $38 \mathrm{I} 4,8^{\star}$ & $6_{4}$ & $-7-4$ \\
\hline \multirow[t]{3}{*}{$74 \ldots \ldots$} & 38 × 3,8 & $38 \mathbf{1} 4, \mathbf{I}$ & $8-6$ & $-7-4$ \\
\hline & - & $3812,8^{\star}$ & $8_{\ddot{z}}$ & -76 \\
\hline & - & $3812,8^{\star}$ & $8_{6}$ & -77 \\
\hline \multirow[t]{5}{*}{$75 \ldots$} & $38 \mathrm{II}, 4$ & 38 II $\star$ & $9-9$ & $-8_{-8}$ \\
\hline & - & 38 го, $9^{\star}$ & $9-9$ & $-8-7$ \\
\hline & - & $3810,8^{\star}$ & $8_{7}$ & -85 \\
\hline & - & $3810,6^{\star}$ & $10-7$ & $-10-9$ \\
\hline & - & $3810,6^{\star}$ & $10-8$ & $-10-10$ \\
\hline $76 \ldots$ & 3809 & - & & - \\
\hline $77 \ldots$ & 3807,6 & 3807,9 & $2_{0}$ & $-I_{1}$ \\
\hline $78 \ldots$ & 3806,8 & $3806,6^{\star}$ & $7-4$ & $-6-4$ \\
\hline \multirow[t]{2}{*}{$79 \ldots \ldots$} & 3804,7 & - & & - \\
\hline & - & 3803,7 & 4-2 & $-3-3$ \\
\hline \multirow[t]{2}{*}{$80 \ldots}$. & 3802,9 & 3802,4 & $3_{2}$ & $-4-3$ \\
\hline & - & 3802,4 & $2_{-1}$ & $-I_{-1}$ \\
\hline
\end{tabular}

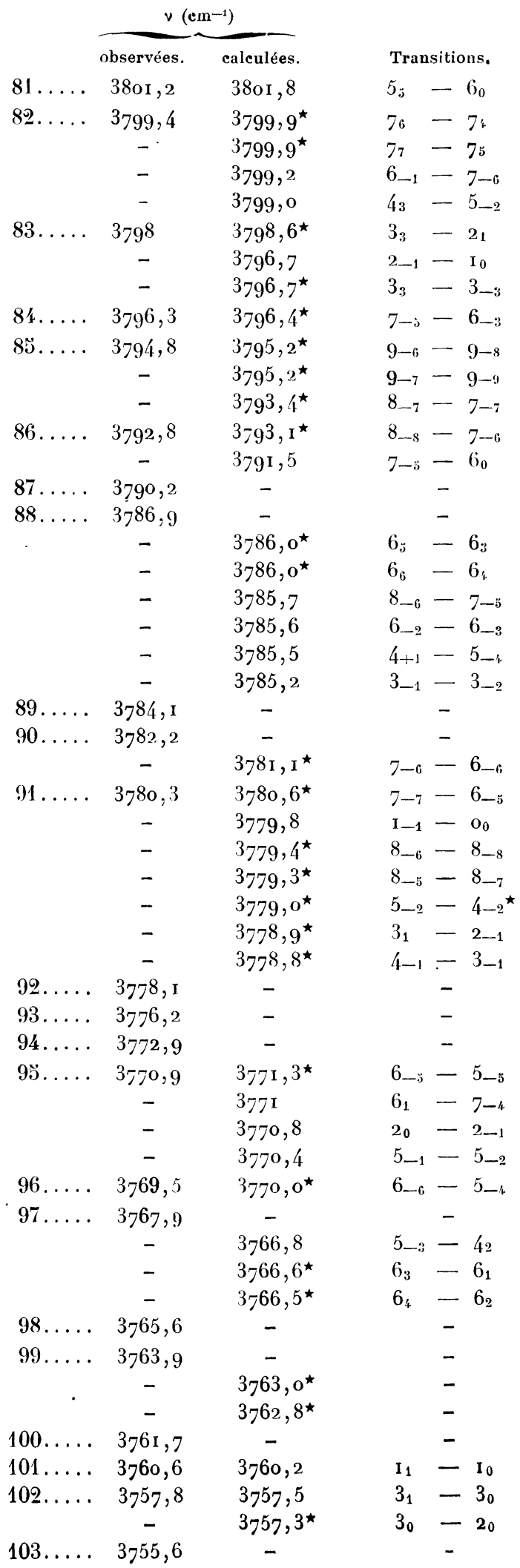


Tableau III (suite).

\begin{tabular}{|c|c|c|c|c|c|c|c|c|c|c|}
\hline \multirow{4}{*}{$104 \ldots}$. & \multicolumn{2}{|c|}{$\nu\left(\mathrm{cm}^{-1}\right)$} & \multirow{3}{*}{\multicolumn{2}{|c|}{$\begin{array}{c}\text { Transitions. } \\
-\end{array}$}} & \multicolumn{3}{|c|}{$\nu\left(\mathrm{cm}^{-1}\right)$} & \multirow{2}{*}{\multicolumn{3}{|c|}{ Transitions. }} \\
\hline & observées. & calculées. & & & \multirow{3}{*}{$\begin{array}{l}124 \ldots \ldots \\
125 \ldots \ldots\end{array}$} & \multirow{3}{*}{$\begin{array}{c}\text { observées. } \\
3719,3 \\
3717,2\end{array}$} & \multirow{2}{*}{$\begin{array}{c}\text { calculées. } \\
3719,4\end{array}$} & & & \\
\hline & 3754,2 & - & & & & & & \multirow{2}{*}{\multicolumn{3}{|c|}{$\begin{array}{c}5_{-2}-5_{-1} \\
-\end{array}$}} \\
\hline & - & 3752,4 & $22_{2}$ & $-2_{1}$ & & & - & & & \\
\hline & - & 3752,3 & $6-_{2}$ & $-7-7$ & $126 \ldots$ & 3716,0 & $37 \mathrm{r} 6, \mathrm{I}^{\star}$ & $5_{0}$ & - & $5-2$ \\
\hline \multirow[t]{4}{*}{$105 \ldots$} & 3751,7 & $375 \mathrm{r}, 9$ & $3_{3}$ & $-4-2$ & & - & 3715,6 & 64 & - & $6_{3}$ \\
\hline & - & 3750,5 & $2_{2}$ & $-3_{3}$ & & - & 3715,6 & $6_{3}$ & - & 64 \\
\hline & - & $3750, x$ & $2_{1}$ & $-2_{2}$ & & - & $3715,6^{\star}$ & $4-2$ & - & $3_{0}$ \\
\hline & - & 3749,8 & $\mathbf{I}_{0}$ & $-\mathfrak{1}_{1}$ & $127 \ldots \ldots$ & $3714, \mathrm{I}$ & - & . & - & \\
\hline $106 \ldots$ & 3749,4 & 3749,7 & $6-6$ & $-5_{-1}$ & & - & 3712,7 & $\mathbf{I}_{0}$ & - & $2-1$ \\
\hline \multirow[t]{3}{*}{$107 \ldots$} & 3748,2 & 3748,3 & $8_{-6}$ & $-9-7$ & & - & $3712,5^{\star}$ & 42 & - & 40 \\
\hline & - & 3747,0 & $8_{-5}$ & $-9-6$ & $128 \ldots$ & 3711,4 & - & & - & \\
\hline & - & $3746,3^{\star}$ & $5-4$ & $-4-4$ & $129 \ldots$ & 3710,1 & $3710, I^{\star}$ & $3_{0}$ & - & $3-2$ \\
\hline \multirow[t]{2}{*}{$108 \ldots \ldots$} & $3746, \mathrm{I}$ & $3746, \mathrm{I}$ & $6_{0}$ & $-6-1$ & & - & $3710,0^{\star}$ & $5-3$ & - & $5_{-5}$ \\
\hline & - & 3745,8 & $5_{1}=$ & $-5_{0}$ & & - & $3709,9^{\star}$ & $3_{2}$ & - & $4-4$ \\
\hline \multirow[t]{3}{*}{$109 \ldots$} & 3745,5 & $3745 ; 4^{\star}$ & $6-4$ & $-6-6$ & & - & 3709,8 & $I_{-1}$ & - & $2-2$ \\
\hline & - & $3745, \mathrm{I}$ & $3_{0}$ & $-3_{1}$ & $130 \ldots$ & 3708,4 & 3708,4 & 7 & - & 75 \\
\hline & - & $3744,6^{\star}$ & 43 & -41 & & - & 3708,4 & 75 & - & 74 \\
\hline \multirow[t]{4}{*}{$110 \ldots$} & 3743,8 & $3743,5^{\star}$ & $2_{2}$ & $-I_{0}$ & & - & $3707, x^{\star}$ & $3-3$ & - & $2-1$ \\
\hline & - & $3743,2^{\star}$ & 44 & -42 & $131 \ldots$ & 3706,8 & 3706,9 & $5_{1}$ & - & $6-4$ \\
\hline & - & 3743,1 & $5_{3}$ & $-6_{-2}$ & $132 \ldots$ & 3705,7 & 3705,6 & $6_{5}$ & - & $6_{6}$ \\
\hline & - & 3742,9 & $4-2$ & $-3_{3}$ & & - & 3705,6 & $6_{6}$ & - & $6_{5}$ \\
\hline \multirow[t]{5}{*}{$111 \ldots$} & 3742,2 & 3741,9 & $3_{1}$ & $-4-4$ & $133 \ldots$ & 3704 & - & & - & \\
\hline & - & 3741,8 & $3_{3}$ & $-3_{2}$ & $134 \ldots$ & 3702,6 & 3702,8 & $4-3$ & - & $4-2$ \\
\hline & - & $3741,8^{\star}$ & $5-5$ & $-4-3$ & & - & $3702,5^{\star}$ & $2-1$ & - & $I_{-1}$ \\
\hline & - & 3741,6 & $3_{2}$ & $-3_{3}$ & & - & $3702,4^{\star}$ & $6-1$ & - & $7-7$ \\
\hline & - & $3741,6^{\star}$ & $6_{1}$ & $-6 b_{-1}$ & & - & 3702,3 & $\mathbf{I}_{1}$ & - & $2_{0}$ \\
\hline $112 \ldots$ & 3740,6 & 3741,5 & 40 & $-5-5$ & $13 \check{.}$ & $370 \mathrm{I}, \mathrm{I}$ & - & & - & \\
\hline $113 \ldots$ & 3738,9 & 3738,8 & $2-1$ & $-2_{0}$ & & - & $3700,0^{\star}$ & 4-2 & - & $4-4$ \\
\hline \multirow[t]{6}{*}{$114 \ldots$} & 3737,2 & $3737, I^{\star}$ & $\mathbf{2}_{1}$ & $-\mathrm{I}_{1}$ & & - & $3700,0^{\star}$ & $2_{1}$ & - & $2-1$ \\
\hline & - & $3736,5^{\star}$ & $4-3$ & $-3_{-3}$ & $136 \ldots$ & 3699,6 & 3699,0 & $5-4$ & - & 41 \\
\hline & - & 3735,7 & $5_{3}$ & $-5_{2}$ & & - & 3699,3 & 70 & - & 77 \\
\hline & - & 3735,7 & $6-1$ & $-6-3$ & & - & 3699,3 & 77 & - & 76 \\
\hline & - & 3735,5 & $4-1$ & -40 & & - & 3698,6 & $6-3$ & - & $6-2$ \\
\hline & - & 3735,5 & $5_{2}$ & $-5_{3}$ & $137 \ldots \ldots$ & 3698,3 & 3698,2 & $5-1$ & - & 44 \\
\hline $115 \ldots$ & 3735,1 & 3735,3 & $6_{2}$ & $-6_{1}$ & $138 \ldots$ & 3697,8 & $3697,9^{\star}$ & 41 & - & $5-5$ \\
\hline & - & 3734,9 & $5-3$ & $-4-1$ & $139 \ldots$ & 3696,2 & $3696,7^{\star}$ & $6_{-2}$ & - & $6-4$ \\
\hline $116 \ldots$ & 3733,1 & 3733 & $\mathrm{o}_{0}$ & $-I_{-1}$ & $140 \ldots$ & 3694,7 & $3695, \mathrm{I}$ & $5_{1}$ & - & $5-1$ \\
\hline & - & $3732,9^{\star}$ & $6_{2}$ & $-6_{0}$ & & - & 3692,7 & $4-3$ & - & $3_{2}$ \\
\hline $117 \ldots$ & $373 \mathrm{I}, 2$ & 373 г, 8 & $7-7$ & $-6_{-2}$ & $141 \ldots$ & 3692 & - & & - & \\
\hline & - & 3730,6 & 42 & -41 & & - & 369 I, 6 & $2-1$ & - & $3-2$ \\
\hline $118 \ldots$ & 3729,2 & $3728,9^{\star}$ & $4-4$ & $-3-2$ & & - & 3691,4 & $2-2$ & - & \\
\hline $119 \ldots$ & 3727,7 & $3727,6^{\star}$ & 43 & $-5_{-3}$ & $142 \ldots$ & 3690,8 & $369 \mathbf{I}, \mathbf{I}$ & $8_{5}$ & - & \\
\hline & - & $3727,4^{\star}$ & $4-1$ & $-4-3$ & & - & $369 \mathrm{I}, \mathrm{I}$ & $8_{6}$ & - & $8_{5}$ \\
\hline & - & 3727,2 & $4 i$ & -43 & & - & $3689,8^{\star}$ & $\mathrm{I}_{0}$ & -- & $o_{0}$ \\
\hline & - & 3726,8 & 43 & -4 & $143 \ldots$ & 3689 & 3689,5 & $2-2$ & - & $3-3$ \\
\hline 120. & 3726,3 & 3726,2 & $5_{0}$ & $-5_{1}$ & & $\cdots$ & $3687,7^{\star}$ & $7-4$ & - & $6_{0}$ \\
\hline & - & $3725,5^{\star}$ & $3_{2}$ & $-3_{0}$ & $144 \ldots \ldots$ & 3686,3 & $3686,3^{\star}$ & $6_{1}$ & - & $7-a$ \\
\hline $121 \ldots \ldots$ & 3724,8 & - & & - & & - & $3686, o^{\star}$ & 40 & - & $4-2$ \\
\hline & - & $3722,8^{\star}$ & 41 & $-4-1$ & & - & $3685,6^{\star}$ & $2_{2}$ & - & $2_{0}$ \\
\hline & - & 3722,8 & $3-2$ & $-3-1$ & & - & 3685,5 & $7-6$ & - & $6-1$ \\
\hline $122 \ldots \ldots$ & 3722,2 & 3722,7 & $6_{1}$ & $-6_{2}$ & & - & 3685,4 & $3-1$ & - & $2_{1}$ \\
\hline & - & $3721,3^{\star}$ & $3_{3}$ & $-3_{1}$ & & - & $3685,4^{\star}$ & $5_{0}$ & - & $6-6$ \\
\hline $123 \ldots$ & 3720,6 & $3721, \mathrm{I}$ & 5 & $-5_{j}$ & & - & 3685,4 & $3-3$ & & $3_{0}$ \\
\hline & - & $372 \mathrm{I}, \mathrm{I}$ & $5_{\ddot{z}}$ & $-5_{4}$ & & - & $3685,0^{\star}$ & $3_{1}$ & - & $3-1$ \\
\hline & - & $3719,9^{\star}$ & $3-2$ & $-2-2$ & & - & $3683,6^{\star}$ & $6_{0}$ & & \\
\hline
\end{tabular}


Tablead III (suite),

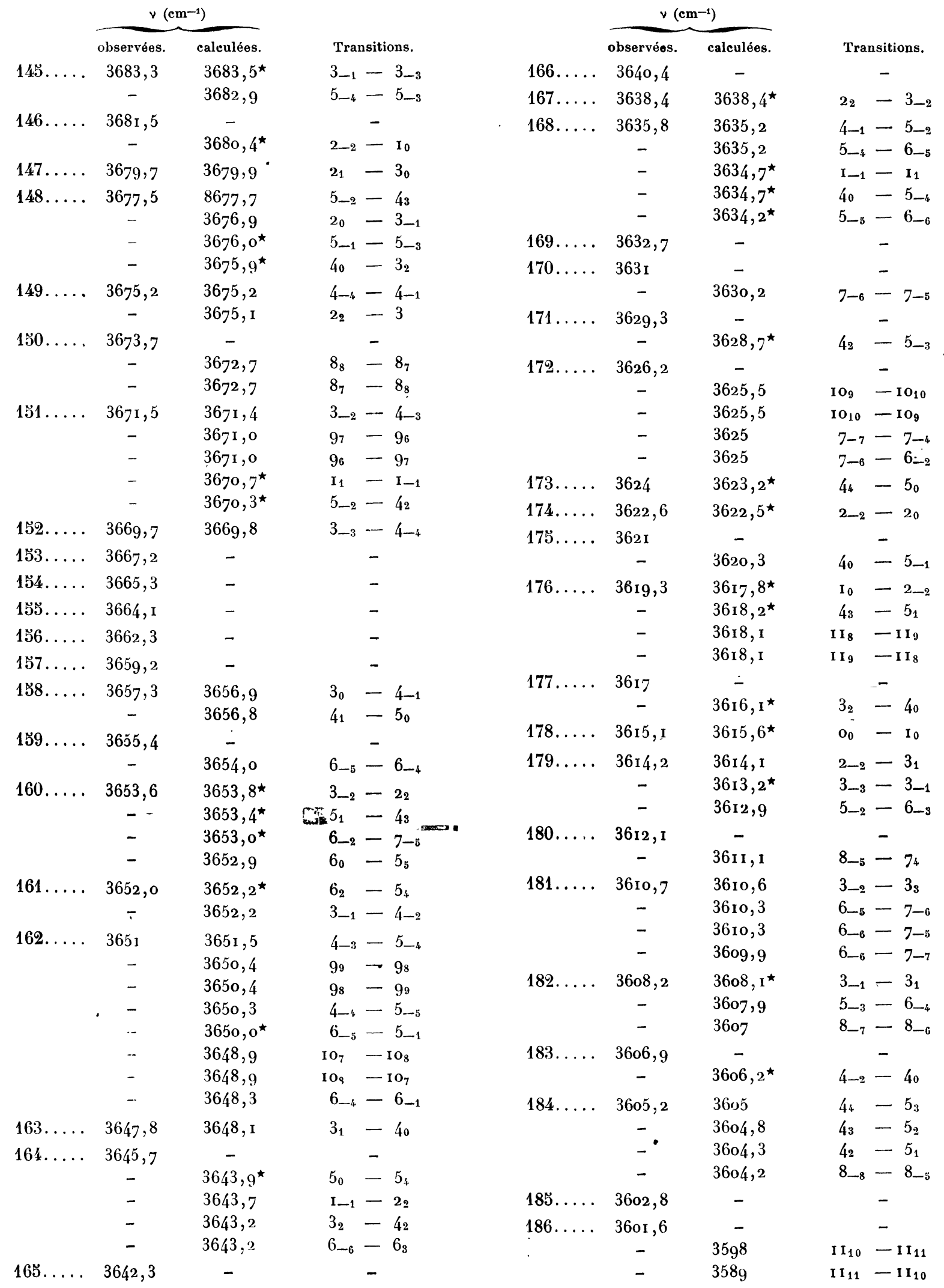


Tableau IV.

\begin{tabular}{|c|c|c|}
\hline$J$. & (1) & \\
\hline$\rho_{0}$. & 5333,5 & + \\
\hline$I_{1} \ldots \ldots$ & 5377,0 & + \\
\hline$I_{0} \ldots \ldots$ & 537 & - \\
\hline$I_{-1} \ldots \ldots$ & 53 & 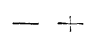 \\
\hline $2 \ldots \ldots$ & 54 & + \\
\hline${ }_{1} \ldots \ldots$ & & - \\
\hline${ }_{0} \ldots \ldots$ & & - \\
\hline $2-1 \ldots \ldots$ & 54 & + \\
\hline${ }_{-2} \ldots$ & $5 / 4$ & + \\
\hline $3 \ldots$ & & + \\
\hline$\beta_{2} \ldots \ldots$ & & - \\
\hline $3_{1} \ldots \ldots$ & & - \\
\hline $3_{0} \ldots \ldots$ & & + \\
\hline $3_{-1} \ldots \ldots$ & & + \\
\hline $3_{-2} \ldots$ & & - \\
\hline $3_{-3} \ldots$ & 5 & \\
\hline 44. & & + \\
\hline 43. & & - \\
\hline $42 \ldots$ & 57 & - \\
\hline 41. & & + \\
\hline 40. & & + \\
\hline $4-1$ & & - \\
\hline $4-2$ & 56 го, & - \\
\hline $4-3$ & - & $\div$ \\
\hline $4-4$ & 5550,2 & + \\
\hline $5_{i j}$. & & -1 \\
\hline & & - \\
\hline $5:$. & 59 & - \\
\hline $5_{2} \ldots \ldots$ & & $e^{-}$ \\
\hline $5_{1} \ldots \ldots$ & 5850,4 & + \\
\hline
\end{tabular}

\begin{tabular}{|c|c|c|c|}
\hline o. & (c1 & & \\
\hline $0 \ldots \ldots$ & 5847,2 & - & - \\
\hline$-1 \ldots$ & 5780,0 & - & $T$ \\
\hline $2 \ldots$ & 5752,2 & + & \\
\hline $3 \ldots$ & 5724,4 & + & \\
\hline$-4 \ldots$ & 5655,6 & - & 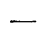 \\
\hline$n \ldots$ & 5653,9 & - & + \\
\hline$\ldots \ldots$ & 6436 & $\perp$ & - \\
\hline$\ldots \ldots$ & $643 c$ & - & - \\
\hline$\ldots \ldots$ & 62.44 & - & - \\
\hline$\ldots \ldots$ & 6244 & + & - \\
\hline$\ldots \ldots$ & 610 & + & + \\
\hline$\ldots \ldots$ & $609^{\circ}$ & - & + \\
\hline$\ldots \ldots$ & 59 & - & - \\
\hline$-1 \ldots \ldots$ & $59^{28,5}$ & + & - \\
\hline$-2 \ldots \ldots$ & 59 & + & + \\
\hline$-3 \ldots \ldots$ & 5860 & - & + \\
\hline$-1 \ldots \ldots$ & 585 & - & - \\
\hline$-\ddot{z} \ldots$ & $5>3$ & + & - \\
\hline$-6 \ldots$ & $5-3$ & + & + \\
\hline . & 679 & + & 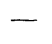 \\
\hline$\ldots \ldots$ & 6798 & - & . \\
\hline$\ldots \ldots$ & 660 & - & - \\
\hline$\ldots \ldots$ & 6602 & + & \\
\hline$\cdots$ & & & \\
\hline$-n \ldots$ & 6081 & + & + \\
\hline$-3 \ldots$ & 6081,0 & + & \\
\hline$-6 \ldots$ & 5883,6 & - & 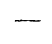 \\
\hline$-7 \ldots$ & 5883,6 & - & -1 \\
\hline & 721 & + & \\
\hline & & - & + \\
\hline$\ldots \ldots$ & 6996,8 & $\rightarrow$ & \\
\hline & 6996,8 & 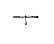 & \\
\hline
\end{tabular}

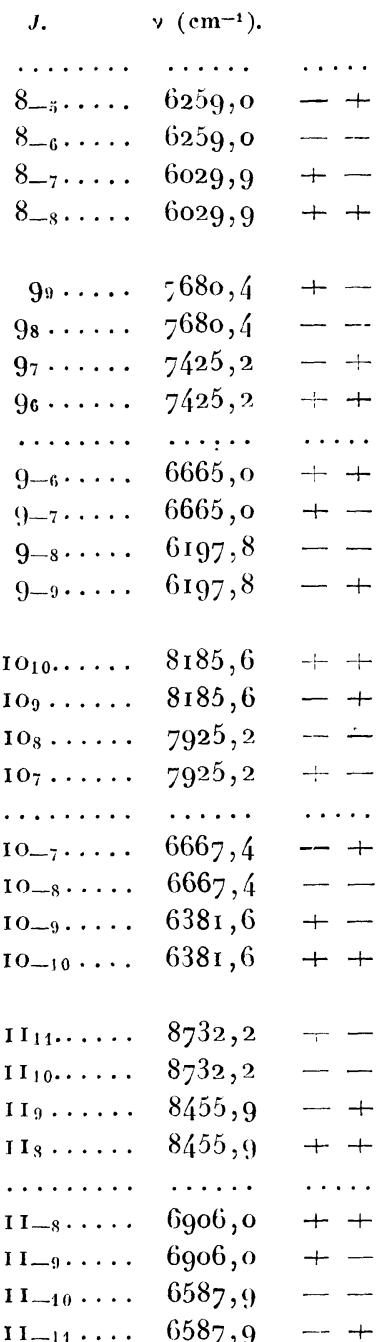

De $J=$ o à $J=6$, les niveaux d'énergie indiqués sont ceux calculés par Nielsen.

Détermination de certaines constantes de la molécule. - La détermination du centre des bandes fournit une valeur expérimentale des fréquences de vibration de ces bandes.

Pour $\nu_{1}+\nu_{3}$, le centre apparaît visiblement sur le spectre entre les bandes 88 et 89 et se situe sensiblement à $5334 \mathrm{~cm}^{-1}$. Pour $\nu_{1}$ et $\nu_{3}$, les centres sont beaucoup plus difficiles à situer du fait du recouvrement des deux bandes, et je ne les ai pas déterminés.

Les constantes de rotation $A, B, C$, de la molécule, ou les moments d'inertie $I_{A}, I_{B}, I_{C}$, ont été redéterminés, pour chacun des trois états de vibrationrotation envisagés, à partir des niveaux de nombre quantique total I et 2, compte tenu de la correction de force centrifuge (faible pour ces niveaux) proposée par Dennison

$$
\begin{aligned}
\mathfrak{1} A & =\frac{h}{3 \pi^{2} c I_{A}}=\left(21-I_{-1}\right) \exp +0,28, \\
4 B & =\frac{h}{2 \pi^{2} c I_{B}}=\left(\begin{array}{ll}
20 & -\mathrm{I}_{0}
\end{array}\right) \exp +0,03, \\
4 & =\frac{h}{2 \pi^{2} c I_{C}}=\left(2-1 \cdot-I_{1}\right) \exp +0,03 ; \\
h & =6,626 \cdot 10^{-27}, \quad c=2,99776 \cdot 10^{10} .
\end{aligned}
$$

A partir de ces valeurs de $I_{A}, I_{B}$ et $I_{C}$, on peut calculer, pour chacun des états vibratoires envisagés, l'angle $\widehat{\mathrm{HOH}}=\alpha$ de la molécule, et la distance $\mathrm{OH}=r$.

$$
\begin{gathered}
\frac{I_{B}}{I_{A}}=1,125 \operatorname{tg}^{2} \frac{\alpha}{2}, \\
I_{C}=2 H r^{2}\left(\mathrm{I}-\frac{\mathrm{I}}{9} \cos ^{2} \frac{\alpha}{2}\right), \\
H=\frac{m_{H}}{N}=\frac{\mathrm{I}, 008}{6,02 \cdot 10^{23}} .
\end{gathered}
$$


Tableau V.

\begin{tabular}{|c|c|c|c|c|}
\hline & \multicolumn{2}{|c|}{$V\left(\mathrm{~cm}^{-1}\right)$} & \multirow{2}{*}{\multicolumn{2}{|c|}{ Transitions. }} \\
\hline & observées. & calculées. & & \\
\hline & $5 \mathrm{I} 5 \mathrm{I}, 4$ & - & & - \\
\hline $1 \ldots$. & - & 5152,2 & $6-4$ & $-7-3$ \\
\hline $2 \ldots$ & 5153,6 & - & & - \\
\hline \multirow[t]{2}{*}{$3 \ldots \ldots$} & 5 I 54,9 & - & & - \\
\hline & - & 5156,8 & $6-3$ & $-7-4$ \\
\hline $4 \ldots$ & 5158,6 & - & & - \\
\hline $5 \ldots \ldots$ & $5 \mathrm{I} 6 \mathrm{I}$ & - & . & - \\
\hline $6 \ldots$ & 5 I 62,6 & - & & - \\
\hline $7 \ldots$ & $5 \mathrm{I} 64,8$ & - & & - \\
\hline $8 \ldots \ldots$ & $5 \mathrm{I} 66, \mathrm{I}$ & 5165,7 & $5-\ddot{3}$ & -4 \\
\hline \multirow[t]{2}{*}{$9 \ldots \ldots$} & 5 I 68,0 & - & & - \\
\hline & - & $5174, \mathrm{I}$ & $7-7$ & $-7-i$ \\
\hline \multirow[t]{5}{*}{$10 \ldots$} & 5174,9 & - & & - \\
\hline & - & 5177,1 & $6-6$ & $--6-3$ \\
\hline & - & 5177,2 & $5-1$ & -6 \\
\hline & - & 5178,3 & $8-5$ & $-9-5$ \\
\hline & - & 5179,2 & $7-6$ & - 7- \\
\hline $11 \ldots$ & 5179,7 & 5179,6 & $8-6$ & $-9-7$ \\
\hline $12 \ldots$ & 5181,3 & $5 \mathrm{I} 8 \mathrm{I}, 6$ & $5-3$ & -6 \\
\hline $13 \ldots$ & 5 I 83,2 & - & & - \\
\hline $14 \ldots \ldots$ & 5 I 84,8 & - & & - \\
\hline $15 \ldots$. & 5 I 86,2 & - & & - \\
\hline \multirow[t]{3}{*}{$16 \ldots$} & 5I 88,9 & 5 I 88,8 & $5_{1}$ & $-6_{0}$ \\
\hline & - & $5 \mathbf{I} 89,3$ & $6-5$ & $-6-4$ \\
\hline & - & $5 \mathrm{I} 92, \mathrm{I}$ & $7-5$ & $-64^{\prime}$ \\
\hline $17 \ldots$ & $5 \mathbf{I} 92,9$ & - & & - \\
\hline \multirow[t]{2}{*}{$18 \ldots \ldots$} & $5 \mathrm{r} 94,5$ & - & & - \\
\hline & - & 5195,2 & $7-4$ & $-8_{-5}$ \\
\hline \multirow[t]{3}{*}{$19 \ldots \ldots$} & $5 \mathrm{Ig} 6,2$ & - & & - \\
\hline & - & 5197,9 & $7-3$ & $-\delta_{-6}$ \\
\hline & - & $5 \operatorname{Ig} 8, \mathbf{I}$ & $5_{0}$ & $-6_{-1}$ \\
\hline $20 \ldots \ldots$ & 5198,9 & $5 \times 99,2$ & $5-2$ & $-6_{-3}$ \\
\hline $21 \ldots$. & $520 \mathrm{I}, 6$ & - & & - \\
\hline $22 \ldots \ldots$ & 5202,4 & - & & - \\
\hline $23 \ldots \ldots$ & $5205, \mathrm{I}$ & - & & - \\
\hline \multirow[t]{3}{*}{$24 \ldots \ldots$} & 5207,8 & 5207,2 & $5-5$ & $-6_{-6}$ \\
\hline & - & 5208,0 & 98 & $-\mathrm{IO}_{7}$ \\
\hline & - & 5208,4 & $5-4$ & $-6_{-5}$ \\
\hline $25 \ldots$. & 5208,9 & 5208,7 & 40 & $-5_{-1}$ \\
\hline \multirow[t]{3}{*}{$26 \ldots$} & 5210,5 & 5210,6 & $4-2$ & $-5_{-3}$ \\
\hline & - & 5210,8 & $\mathrm{IO}_{9}$ & $-\mathrm{II}_{8}$ \\
\hline & - & 5210,8 & $1 O_{10}$ & $-I_{I_{9}}$ \\
\hline $27 \ldots \ldots$ & 5211,6 & - & & - \\
\hline \multirow[t]{2}{*}{$28 \ldots \ldots$} & 5214,0 & $52 \mathrm{I} 4, \mathrm{I}$ & $6_{3}$ & -7 \\
\hline & - & $52 \mathrm{I} 4, \mathrm{I}$ & $6_{6}$ & $-7_{\ddot{a}}$ \\
\hline $29 \ldots \ldots$ & $52 \mathbf{I} 6,8$ & - & & - \\
\hline \multirow[t]{6}{*}{$30 \ldots$} & 5219,2 & $52 \mathrm{I} 9, \mathrm{o}$ & 42 & $-5_{1}$ \\
\hline & - & 5219,7 & $5_{4}$ & $-6_{3}$ \\
\hline & - & 5219,8 & $5_{4}$ & $-6_{4}$ \\
\hline & - & $5220, \mathrm{I}$ & $4-1$ & $-5_{-2}$ \\
\hline & - & 5220,5 & $5_{-3}$ & $-5_{0}$ \\
\hline & - & 5220,6 & $I_{-1}$ & $-2_{2}$ \\
\hline
\end{tabular}

\begin{tabular}{|c|c|c|c|c|c|}
\hline & \multicolumn{2}{|c|}{$\nu\left(\mathrm{cm}^{-1}\right)$} & \multirow{2}{*}{\multicolumn{3}{|c|}{ Transitions. }} \\
\hline & observécs. & calculées. & & & \\
\hline $31 \ldots$ & 5221,7 & - & & - & \\
\hline $32 \ldots$ & 5223,8 & 5224,0 & 41 & - & $5_{0}$ \\
\hline $33 \ldots$ & 5225,5 & 5225,0 & $4-4$ & - & $5-5$ \\
\hline \multirow[t]{2}{*}{$34 \ldots$} & 5226,8 & - & & - & \\
\hline & - & 5227,6 & $4-2$ & - & 41 \\
\hline $3 \check{.} . \ldots$ & $5230, \mathrm{I}$ & - & & - & \\
\hline \multirow[t]{2}{*}{$36 \ldots$} & 5232,3 & 5232,0 & $3-1$ & - & $4-2$ \\
\hline & - & 5232,2 & $3_{1}$ & - & 40 \\
\hline \multirow[t]{3}{*}{$37 \ldots$} & 5233,4 & 5233,5 & 44 & - & $5_{3}$ \\
\hline & - & 5233,7 & 43 & - & $5_{2}$ \\
\hline & - & 5234,5 & $7-6$ & - & $6-1$ \\
\hline $38 \ldots \ldots$ & 5236,4 & - & & - & \\
\hline $39 \ldots \ldots$ & 5237,8 & - & & - & \\
\hline $40 \ldots$ & 5239,2 & - & & - & \\
\hline $41 \ldots$ & 5240,8 & 5240,4 & $3_{0}$ & - & $4-1$ \\
\hline $42 \ldots$ & 5243,6 & - & & - & \\
\hline $43 \ldots$. & 5244,9 & - & & - & \\
\hline $44 \ldots$. & 5246,6 & 5246,7 & $3:$ & - & 42 \\
\hline \multirow[t]{2}{*}{$43 \ldots$} & $5248, o$ & 5247,7 & 3_:3 & - & $4-4$ \\
\hline & - & $5248, \mathrm{I}$ & $3_{2}$ & - & 41 \\
\hline $46 \ldots$ & 5249,3 & 5249,9 & 4-: & - & $4-1$ \\
\hline $47 \ldots$ & 5250,4 & 5250,5 & $3-2$ & - & $4-3$ \\
\hline $48 \ldots$ & 53,2 & - & & - & \\
\hline \multirow[t]{3}{*}{$49 \ldots$} & 55,4 & - & & - & \\
\hline & - & $5256, \mathrm{I}$ & $5-4$ & - & $5-3$ \\
\hline & - & 5256,9 & $2_{0}$ & - & $3-1$ \\
\hline$\breve{3} 0 \ldots$. & 5257,6 & - & & - & \\
\hline$\ddot{\partial 1} 1 \ldots .$. & 5259,6 & - & & - & \\
\hline \multirow[t]{3}{*}{$52 \ldots$} & $5262, \mathrm{I}$ & - & & - & \\
\hline & - & 5263,2 & 21 & - & $3_{0}$ \\
\hline & - & 5263,3 & $3-3$ & - & $3_{0}$ \\
\hline \multirow[t]{2}{*}{$53 . \ldots$} & 5264,3 & $5266^{\prime}, \mathrm{o}$ & $5-2$ & - & 43 \\
\hline & - & 5265,9 & $2-2$ & - & $3-3$ \\
\hline $54 \ldots \ldots$ & 5267 & - & & - & \\
\hline $55 \ldots .$. & 5268,4 & 5267,8 & $2-2$ & - & $2_{1}$ \\
\hline \multirow[t]{2}{*}{$56 \ldots}$. & 5270,0 & - & & - & \\
\hline & - & 5270,9 & $6_{0}$ & - & $7-5$ \\
\hline $57 \ldots$ & 5272,9 & - & & - & \\
\hline $58 \ldots$. & 5273,7 & - & & - & \\
\hline $59 \ldots \ldots$ & 5276,5 & - & & - & \\
\hline $60 \ldots$ & 5278,2 & - & & - & \\
\hline \multirow[t]{2}{*}{$61 \ldots$} & 5279,3 & - & & - & \\
\hline & - & 5280,8 & $7-7$ & - & 6 -2 \\
\hline $62 \ldots$ & $5282, \mathrm{I}$ & $5282, \mathrm{o}$ & $I_{1}$ & - & $2_{0}$ \\
\hline $63 \ldots \ldots$ & 5283,5 & - & & - & \\
\hline $64 \ldots$ & 5286 & - & & - & \\
\hline $65 \ldots$ & 5286,8 & 5286,7 & $I_{-1}$ & - & $2-2$ \\
\hline $66 \ldots$ & 5288,8 & - & & - & \\
\hline \multirow[t]{2}{*}{$67 \ldots$} & 5290,7 & - & & - & \\
\hline & - & $529^{2}, 0$ & $\mathrm{I}_{0}$ & - & $2-1$ \\
\hline $68 \ldots$ & 5293,5 & - & & - & \\
\hline $69 \ldots$ & 5296,3 & - & & - & \\
\hline
\end{tabular}


Tableau V (suite).

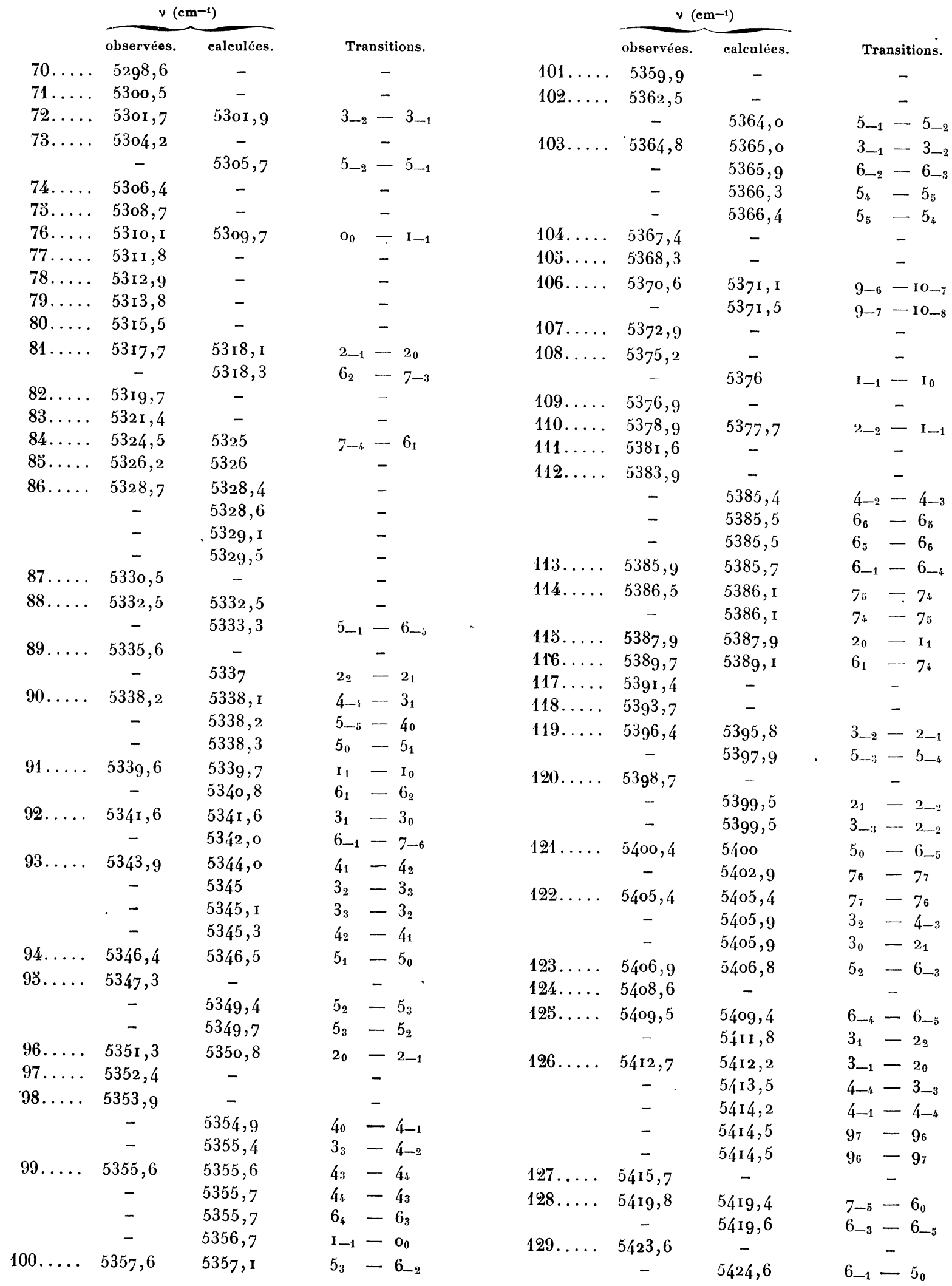


Tableau V (suite).

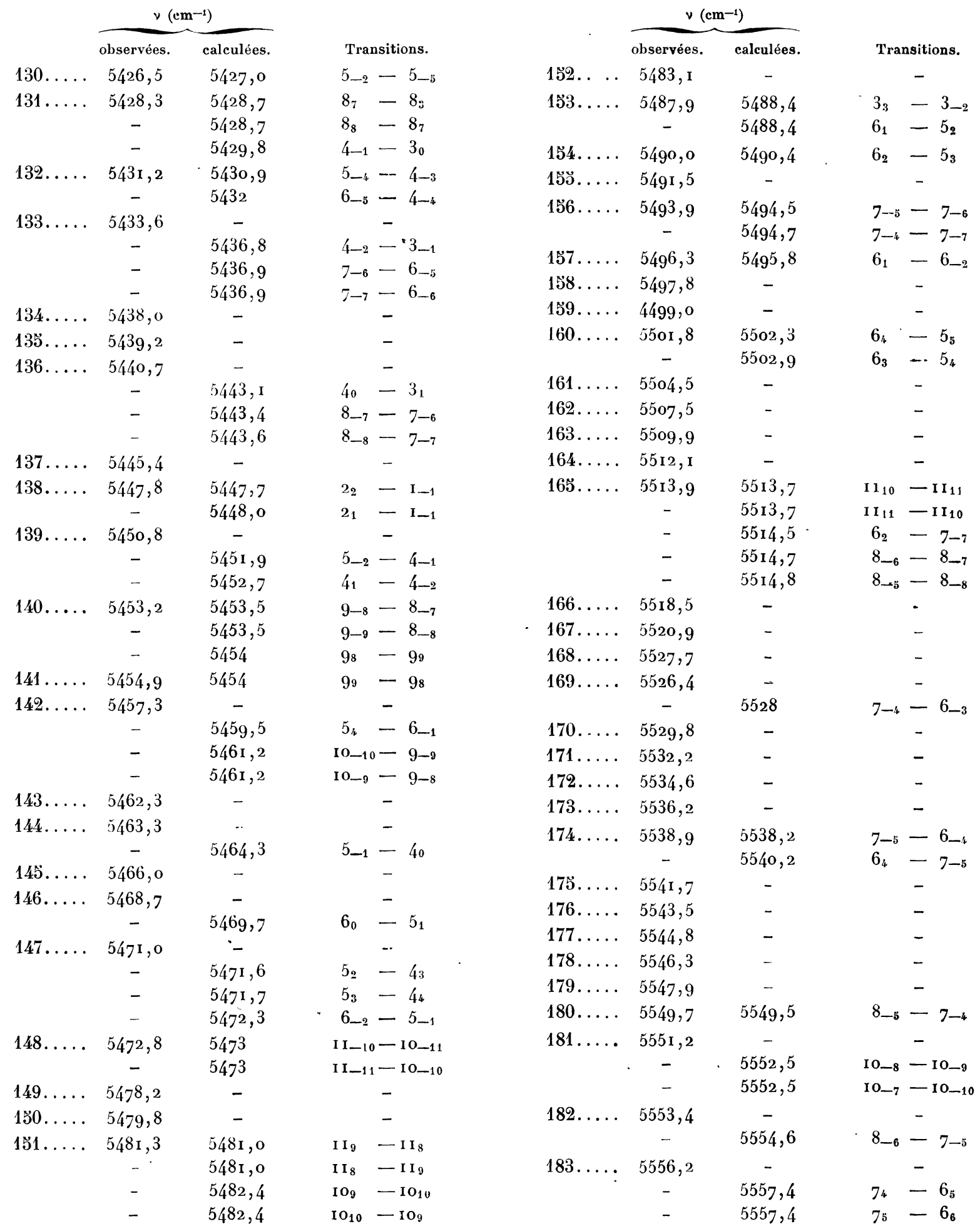

Les résultats obtenus sont rassemblés dans le tableau VI.
Ce travail a été fait, grâce aux crédits fournis par le Centre National de la Recherche scientifique, 


\begin{tabular}{|c|c|c|c|}
\hline$\Lambda \ldots \ldots \ldots \ldots$ & \multicolumn{3}{|c|}{$\nu_{1}(1,0,0)$} \\
\hline B.......... & I 4,39 & $"$ & \\
\hline C. . & 9,06 & ") & \\
\hline$I_{\mathrm{A}} \ldots \ldots \ldots$ & I, Io. & $10^{-20}$ & $\mathrm{~g}: \mathrm{cm}^{3}$ \\
\hline$I_{\mathrm{B}} \ldots \ldots \ldots \ldots$ & I, 95 & $c$ & i \\
\hline $\boldsymbol{I}_{\mathrm{C}} \ldots \ldots \ldots$ & 3,09 & , & ) \\
\hline$r \ldots \ldots \ldots \ldots$ & 0,995 & & \\
\hline$\alpha \ldots \ldots \ldots \ldots$ & $102^{\circ} \mathrm{g}$ & & \\
\hline
\end{tabular}

Tableau VI.

\begin{tabular}{|c|c|c|}
\hline \multicolumn{3}{|c|}{$\begin{array}{r}v_{3}(0,0, \\
26,46 \mathrm{~cm}^{-1}\end{array}$} \\
\hline I 4,58 & $"$ & \\
\hline 8,98 & $"$ & \\
\hline I, 06. & $10^{-i 0}$ & $\mathrm{~g}: \mathrm{cm}^{3}$ \\
\hline I , 92 & & $"$ \\
\hline $3, \mathbf{I} \mathbf{I}$ & & ") \\
\hline $\begin{array}{l}0,9^{8} \mathbf{I} \\
\text { roI }\end{array}$ & & \\
\hline
\end{tabular}

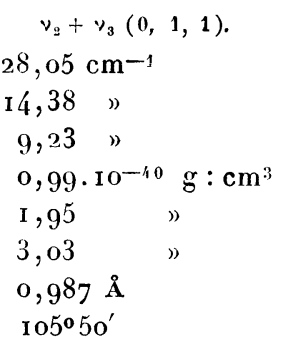

sous la haute direction de M. J. Lecomte, à. qui j'exprime ma gratitude. chewitz, pour les conseils qu'il m'a donnés et l'aide qu'il m'a apportée.

J'adresse mes vifs remerciements à M. P. Bar-

\section{BIBLIOGRAPHIE.}

[1] Sleator W. W. - Astroph. J., i 9 I 8, 48, I 25.

[2] Sleator W. W. et Phelps E. R. - Astroph. J., i 925 , 62,28 .

[3] Phyler E. K. et Sleator W. - Phys. Rev., ig3i, 37, 1433.

[4] Bonner L. G. - Phys. Rev., I 934, 46, 485.

[5] Mecke R. - Z. Physik, 1933, 81, 3 I 3 .

[6] Randall H. M., Dennison D. M., Ginsburg N. et Weber L. - Phys. Rev., I 937,52 , 60.

[7] Darling B. T. et Dennison D. H. - Phys. Rev., 1940, 55, 128.

[8] Shaffer W. H. et Nielsen H. H. - Phys. Rev., r 939 , 56, I 88 .

[9] Nielsen H. H. - Phys. Rev., i 94r, 59, 565.

[10] Nielsen H. H. - Phys. Rev., i $942,62,422$.

[11] Mathis R., Bosson F., Gauthier G. et Larnaudie M. - A paraitre au Journal de Physique.

[12] Sutherland G. B., Blackwell D. E. et Fellget P. B. - Nature, i $946,158,873$.
[13] Nelson R. C. et Benedict W. S. - Phys. Rev., 1948, 74, 702 .

[14] Nelson R. C. - J. Opt. Soc. Amer., x 949, 39, 68.

[15] Witmer E. E. - Proc. Nat. Acad. Amer., i $927,13,60$.

[16] Wang S. C. - Phys. Rev., I929, 34, 243.

[17] Kramers H. A. et Ittmann G. P. - Z. Physik, i925, 53, 553.

[18] Kramers H. A. et Ittmann G. P. - Z. Physik, i929, 58, 2 1 $7 ; 1930,60,663$

[19] Klein O. - Z. Physik, i $929,58,730$.

[20] Rox B. S. - Z. Physik, r $932,78,74$.

[21] King G. W., Hainer R. M. et Gross P. C. - J. Chem. Phys., 1943, 11, 27.

[22] Dennison D. M. - Rev. Mod. Physics, 1931, 3, 280.

[23] Herzberg G. - Molecular spectra and Molecular Structure. II. Infrared and Raman spectra of Polyatomic molecules (D. Van Nostrand Company, New-York). 